\title{
Pinch-off of non-axisymmetric vortex rings
}

\author{
Clara O’Farrell ${ }^{1, \dagger}$ and John O. Dabiri ${ }^{2}$ \\ ${ }^{1}$ Control and Dynamical Systems, California Institute of Technology, Pasadena, CA 91125, USA \\ ${ }^{2}$ Graduate Aeronautical Laboratories and Bioengineering, California Institute of Technology, \\ Pasadena, CA 91125, USA
}

(Received 15 April 2013; revised 29 September 2013; accepted 29 November 2013; first published online 10 January 2014)

The formation and pinch-off of non-axisymmetric vortex rings is considered experimentally. Vortex rings are generated using a non-circular piston-cylinder arrangement, and the resulting velocity fields are measured using digital particle image velocimetry. Three different nozzle geometries are considered: an elliptical nozzle with an aspect ratio of two, an elliptical nozzle with an aspect ratio of four and an oval nozzle constructed from tangent circular arcs. The formation of vortices from the three nozzles is analysed by means of the vorticity and circulation, as well as by investigation of the Lagrangian coherent structures in the flow. The results indicate that, in all three nozzles, the maximum circulation the vortex can attain is determined by the equivalent diameter of the nozzle: the diameter of a circular nozzle of identical cross-sectional area. In addition, the time at which the vortex rings pinch off is found to be constant along the nozzle contours, and independent of relative variations in the local curvature. A formation number for this class of vortex rings is defined based on the equivalent diameter of the nozzle, and the formation number for vortex rings of the three geometries considered is found to lie in the range of 3-4. The implications of the relative shape and local curvature independence of the formation number on the study and modelling of naturally occurring vortex rings such as those that appear in biological flows is discussed.

Key words: biological fluid dynamics, vortex flows, wakes/jets

\section{Introduction}

The formation of axisymmetric vortex rings by the ejection of fluid through a circular aperture has been the subject of several experimental investigations (Maxworthy 1972; Didden 1979; Glezer \& Coles 1990; Gharib, Rambod \& Shariff 1998; Dabiri \& Gharib 2004a). In these studies, a fluid column of length $L$ is ejected through a circular aperture or nozzle of diameter $D$, by the action of a piston moving with prescribed velocity inside of a hollow circular cylinder. Gharib et al. (1998) found that such vortex rings cannot grow indefinitely, but rather there is a physical limit to their size. When the non-dimensional formation time $\hat{T}=\bar{U}_{p} t / D$ (where $\bar{U}_{p}$ is the mean piston velocity and $D$ the nozzle diameter) exceeds approximately four, the vortex rings reject additional vorticity flux and a trailing jet forms behind them. This 
transition, termed 'vortex pinch-off' by Gharib et al. (1998), is predicted by an energy maximization argument due to Kelvin (1880) and Benjamin (1976).

The universality of the formation number has been verified by a number of subsequent experimental (Dabiri \& Gharib 2004a; Krueger, Dabiri \& Gharib 2006; Pawlak et al. 2007) and computational studies (Rosenfeld, Rambod \& Gharib 1998; Mohseni, Ran \& Colonius 2000). In particular, Rosenfeld et al. (1998), Zhao, Frankel \& Mongeau (2000) and Mohseni et al. (2000), found the Reynolds number to have little impact on the formation number, provided that $R e_{\Gamma}$ is sufficiently large to ensure the formation process is completed before viscosity has a significant impact $\left(R e_{\Gamma}>2000\right.$ according to Mohseni et al. (2000)). The concept of the universal formation number has also been extended to more complex vortex generator setups and computational frameworks, by extending the definition of the dimensionless vortex formation time to allow for variations such as the presence of bulk coflow (Krueger et al. 2006) or bulk counterflow (Dabiri \& Gharib 2004a), a time-varying exit diameter (Mohseni et al. 2000; Dabiri \& Gharib 2005), and differing velocity programs (Rosenfeld et al. 1998; Mohseni et al. 2000; Krueger \& Gharib 2003; Olcay \& Krueger 2010) and velocity profiles (Rosenfeld et al. 1998).

The existence of this constraint on vortex ring growth has powerful implications for biological jetting flows, since vortex rings provide a propulsive advantage due to their entrainment of ambient fluid (Auerbach 1991; Dabiri \& Gharib 2004b; Olcay \& Krueger 2007; Shadden et al. 2007) and to the added mass of non-entrained fluid that must be accelerated with the vortex ring (Baird, Wairegi \& Loo 1977; Weihs 1977; Krueger \& Gharib 2003). A constraint on the growth of vortex rings therefore places a limit on the performance of biological jetting flows. For example, Krueger \& Gharib (2003) showed that the normalized time-averaged thrust per pulse in pulsed jetting flows reaches a maximum just after the limiting formation time $\hat{T}=4$. This implies that there is an 'optimal' $\hat{T}$ for jetting propulsion. In fact, recent studies have found evidence of 'optimal vortex formation' in jetting swimmers such as juvenile squid (Bartol et al. 2009) and certain species of jellyfish (Linden \& Turner 2004; Dabiri et al. 2010), and in blood flow in the cardiac left ventricle (Dabiri \& Gharib 2005; Gharib et al. 2006).

However, naturally occurring vortices are rarely circular, and axisymmetric flows such as those generated by squid or jellyfish, are scarce in nature. In fact, most swimming and flying animals generate deformed loop-like vortices rather than circular vortex rings (Kokshaysky 1979; Rayner 1979; Dickinson \& Götz 1996; Kern \& Koumoutsakos 2006; Kim \& Gharib 2011). Similarly, the mitral valve in humans is not axisymmetric, so circular vortex rings do not describe the flow in the human left ventricle with complete accuracy (Bellhouse 1972; Reul, Talukder \& Muller 1981; Wieting \& Stripling 1984; Domenichini, Pedrizzetti \& Baccani 2005). The departure from axisymmetry of most vortex rings of biological relevance is of importance because the limit on vortex ring growth imposed by the Kelvin-Benjamin principle has only been shown to apply to axisymmetric jetting flows. In other, non-axisymmetric, vortex-shedding configurations, no limit on the growth or size of vortices is known. In the case of vortex formation from two-dimensional orifices, for example, recent experimental (Afanasyev 2006) and computational (Pedrizzetti 2010) results suggest that there is no limit on the growth of a vortex dipole.

In this regard, the work of Domenichini (2011) is of particular interest. Domenichini (2011) studied the formation of vortices from slender orifices whose outline consisted of two semicircular portions connected by two rectilinear segments of varying length. 
In his computations, the author found that the roll-up of the shear layer and vortex formation process differed considerably on the circular and rectilinear segments. In particular, for nozzles with rectilinear segments of sufficient length, he found evidence of pinch-off at the centre of the circular portions at a non-dimensional time consistent with the work of Gharib et al. (1998), but no evidence of pinch-off at the centre of the rectilinear portions. The author attributed these differences to the dependence of the self-induced velocity of the lead vortex ring on local curvature (Arms \& Hama 1965; Leonard 1985; Saffman 1992), which caused the portions of the vortex closest to the circular sides of the slender orifices to move away from the shear layer faster than the portions near the rectilinear sides. Although Helmholtz's laws dictate the overall geometry of the vortex formation process, they do not preclude pinch-off from happening at different times if the vortex filament geometry is complex. Hence, Domenichini (2011) concluded that pinch-off in these non-axisymmetric vortex rings can be a local phenomenon, determined by local curvature.

In his concluding remarks, Domenichini (2011) theorized that his findings could, with small modifications, extend to other non-axisymmetric geometries in general, and elliptical vortex rings in particular. However, while the behaviours of isolated noncircular vortex rings and of non-axisymmetric jets have been the subject of extensive study, the vortex formation process for non-axisymmetric rings has not been analysed in detail. Hence, Domenichini's conjecture remains untested.

The evolution of isolated vortex rings of non-circular shape has been examined in a variety of computational, analytical and experimental investigations. In particular, the behaviour of elliptical vortex rings is well documented in both computational (Arms \& Hama 1965; Viets \& Sforza 1972; Dhanak \& de Bernardinis 1981; Fernandez, Zabusky \& Gryanik 1995; Ryu \& Lee 1997; Kimura 2006) and experimental studies (Oshima et al. 1988; Hussain \& Husain 1989; Hussain \& Hussain 1991; Adhikari 2009). The bulk of these studies focused on the time-dependent deformation of the elliptical vortex ring due to the curvature dependence of the vortex propagation velocity (Arms \& Hama 1965; Dhanak \& de Bernardinis 1981; Viets \& Sforza 1972). Similar experimental and numerical methods have been applied in studies of the deformation of isolated vortex rings with other non-circular geometries, including those with initially rectangular (Viets \& Sforza 1972; Grinstein 1995; Kambe \& Takao 1971) and lenticular (Kambe \& Takao 1971; Oshima 1972) shapes. Viets \& Sforza (1972) also considered the evolution of a vortex ring whose initial shape was described by a superellipse or Lamé curve. It is interesting to note that Kiya \& Ishii (1991) and Kiya et al. (1992) characterized the deformation of isolated 'pseudo-elliptic' vortex rings of a shape identical to those considered by Domenichini (2011).

Considerable attention has also been devoted to the study of non-circular free jets, much of it motivated by their favourable mixing properties and reduced jet noise when compared to circular jets (Ho \& Gutmark 1987; Ahuja, Manes \& Massey 1990; Gutmark \& Grinstein 1999). Since the dynamics of the non-circular vortex rings in these jets are thought to be the driving force behind these beneficial properties, numerous studies on non-circular jets have focused on the dynamics of the vortex rings in the jet. In particular, Husain and Hussain (Hussain \& Husain 1989; Hussain \& Hussain 1991; Husain \& Hussain 1993) conducted a comprehensive study of the dynamics of vortex rings in elliptic jets. A similarly detailed treatment of the dynamics of vortex rings in rectangular jets is found in the works of Grinstein, Gutmark and others (Grinstein, Glauser \& George 1995; Gutmark \& Grinstein 1999; Grinstein 2001). 
Except for the work of Adhikari (2009), however, all of the aforementioned studies focused on either isolated vortex rings at low stroke ratios or on the dynamics of vortices in a steady jet. Adhikari (2009) considered the effect of increasing stroke length on the formation of elliptical vortex rings, and compared the interaction of the growing vortex ring with its trailing shear layer to the dynamics and pairing of vortex rings in elliptic jets as described in Husain and Hussain's study (Hussain \& Husain 1989; Hussain \& Hussain 1991; Husain \& Hussain 1993). In addition, Adhikari (2009) conducted the only experimental study of non-circular vortex rings using digital particle image velocimetry (DPIV) to date. However, the author did not address the existence of any limits on the size of the vortex ring, nor did he compute a formation number analogous to that of Gharib et al. (1998) or consider the dependence of the vortex formation on curvature. As a result, the relationship of Adhikari's vortex rings to Domenichini's conjecture remains unknown.

In this study, we considered the formation of non-axisymmetric vortex rings at long stroke ratios, as well as the effect of curvature on the vortex formation process and on the maximum size of the vortex ring. The present investigations aim to bridge the gap in our current understanding of non-circular vortex rings, by considering the intermediate states lying between isolated non-axisymmetric vortex rings and steady non-circular jets. In particular, we tested Domenichini's hypothesis in a wider class of non-circular vortex rings, in order to determine what role local and global parameters play in the onset of pinch-off in non-circular starting jets. Ultimately, the goal of this study was to define an analogue to the formation number of Gharib et al. (1998), for the development of vortex rings with moderate departure from axisymmetry of relevance to biological flows. To this end, we conducted an experimental study of the formation of vortex rings from three non-circular nozzles: two elliptical nozzles with aspect ratios of two and four, respectively, and an oval nozzle constructed from two pairs of tangent circular arcs.

In addition to classical Eulerian techniques, we visualized the non-circular vortices by means of the Lagrangian coherent structures (LCS) in the flow. LCS are finitetime analogues to the stable and unstable manifolds, for systems with arbitrary time dependence. LCS were introduced in a series of papers by Haller and coworkers (Haller \& Poje 1998; Haller 2000, 2001a,b, 2002; Haller \& Yuan 2000), and have been employed for structure identification and for investigating vortex dynamics by Shadden, Dabiri \& Marsden (2006) and Shadden et al. (2007), Green, Rowley \& Haller (2007) and Green, Rowley \& Smits (2010), O'Farrell \& Dabiri (2010) and others.

The results of the Eulerian and Lagrangian analyses suggested that, for vortex rings with a moderate deviation from axisymmetry such as those found in biological flows, global parameters dominate the formation process. Hence, a non-dimensional critical formation time, or formation number, for this class of vortex rings was defined based on the equivalent diameter of the nozzle (the diameter of a circular nozzle with equivalent cross-sectional area).

The paper is organized as follows. In $\S 2$ we describe the experimental set-up and methods utilized to collect planar velocity data on the non-circular nozzles, as well as the numerical methods used to compute the LCS. The formation and later deformation of the vortex rings are described in $\S \S 3$ and 4, from the Eulerian and Lagrangian perspectives, respectively. The effect of curvature on the growth and limiting formation number of non-axisymmetric rings is examined in $\S 5$. Finally, a comparison of our results with those of Domenichini (2011), and their implications to the study of biologically relevant vortex flows are considered in $\S 6$. 


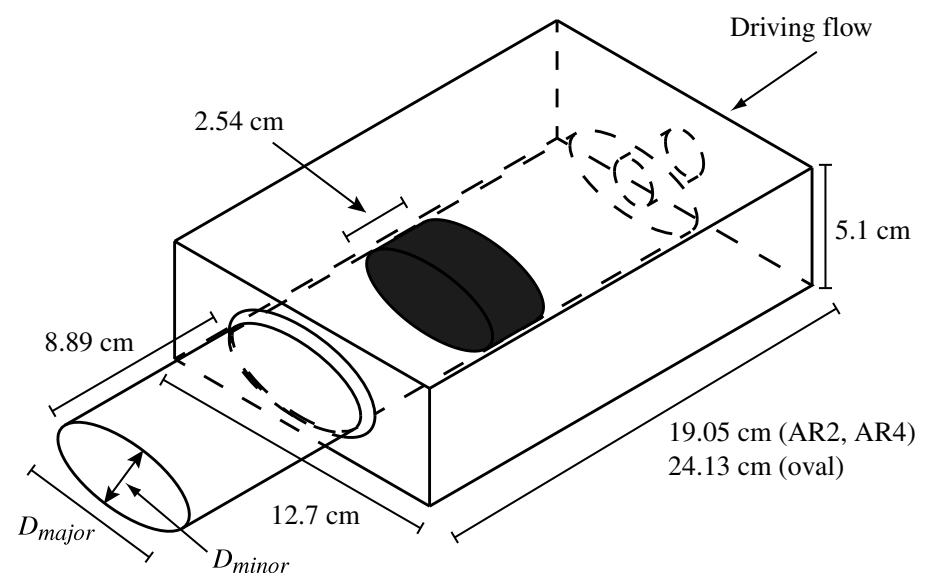

FIGURE 1. Vortex ring generator geometry and dimensions. The vortex generator consisted of an acrylic block attached to a non-circular nozzle with one of three cross-sectional shapes: an ellipse with an aspect ratio of two, an ellipse with an aspect ratio of four and an oval constructed from tangent circular segments of constant curvature. The dimensions of the major and minor axes for the three nozzles considered are listed in table 1.

\section{Methods}

\subsection{Experimental methods}

We considered the formation of non-axisymmetric vortex rings from three nozzles of different cross-sectional shape: an elliptical nozzle with an aspect ratio of two, an elliptical nozzle with an aspect ratio of four and an oval nozzle constructed from four connected segments with constant, non-zero curvature. These nozzles will henceforth be referred to as the AR2 nozzle, the AR4 nozzle and the oval nozzle, respectively. The three vortex generator devices consisted of an acrylic block culminating in a sharp-edged nozzle with a cross-section in one of the three aforementioned shapes (figure 1). A hollow cavity with the same cross-sectional shape as the nozzle ran the length of the device, and an identically shaped piston was allowed to slide freely along the cavity.

A summary of the geometrical properties of the three nozzle shapes considered is given in table 1. The three nozzle shapes were selected to encompass a wide class of those non-circular nozzles not considered by Domenichini (2011). In the two elliptical nozzles, the curvature varied continuously from its minimum value at the minor axis of the ellipse, to its maximum value at the major axis. However, the range of curvatures was much larger in the AR4 nozzle than in the AR2 nozzle $\left(\kappa_{\max } / \kappa_{\min } \approx 60\right.$ and $\approx 8$, respectively). This allowed us to assess the importance of relative variations in the local curvature and global parameters to pinch-off, and to ascertain whether large variations in curvature were required to produce the spatially varying results reported by Domenichini (2011).

However, the nozzles considered by Domenichini (2011) did not exhibit a smooth variation in the curvature, but rather they contained discontinuities in their curvature where the straight segments met the circular segments. Furthermore, the curvature on the rectilinear segments of Domenichini's nozzles was zero, while the elliptical nozzles described above did not include regions of zero curvature. In order to ascertain whether discontinuities in curvature are required to produce the spatially varying 


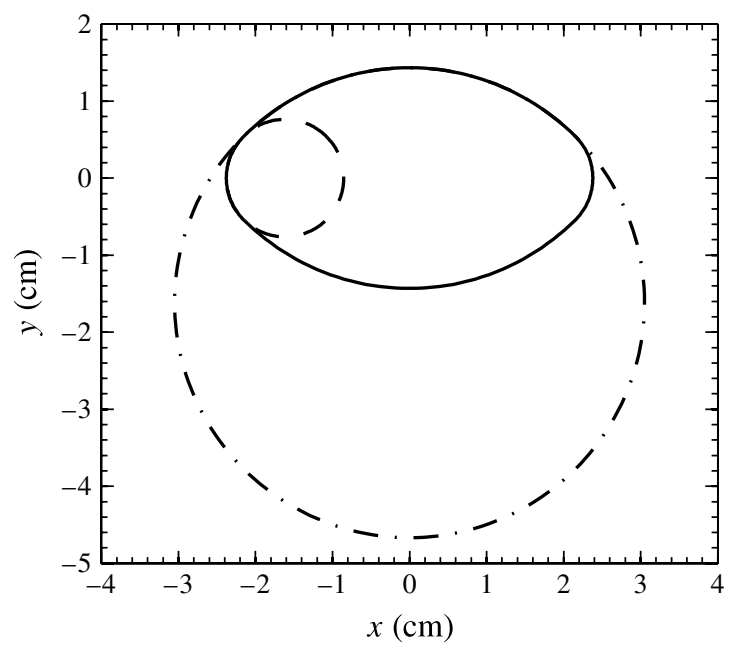

FIgURE 2. Cross-section of the oval nozzle. The two sections closest to the $x$-axis are tangent to a circle of radius $R=0.8 \mathrm{~cm}(--)$ and the two sections nearest to the $y$-axis are tangent to a circle of radius $R=3.1 \mathrm{~cm}(-\cdot-)$.

$\begin{array}{lccc}\text { Dimension } & \text { AR2 ellipse } & \text { AR4 ellipse } & \text { Oval nozzle } \\ D_{\text {eq }}(\mathrm{cm}) & 4.45 & 4.45 & 3.62 \\ D_{\text {major }}(\mathrm{cm}) & 6.35 & 8.89 & 4.76 \\ D_{\text {minor }}(\mathrm{cm}) & 3.17 & 2.22 & 2.86 \\ \kappa_{\text {min }}\left(\mathrm{cm}^{-1}\right) & 0.16 & 0.06 & 0.33 \\ \kappa_{\text {max }}\left(\mathrm{cm}^{-1}\right) & 1.26 & 3.6 & 1.31 \\ \text { Maximum piston stroke } & 21.6 & 21.6 & 28.2 \\ (\mathrm{~cm}) & & & \end{array}$

TABLE 1. Shape and dimensions of the three non-axisymmetric nozzle shapes considered.

pinch-off observed by Domenichini (2011), we constructed an oval nozzle from the intersection of two pairs of circular segments with distinct but non-zero curvature, which resulted in discontinuities in the curvature at the intersections of the segments (see figure 2).

The vortex generators were submerged in a water tank and vortex rings were generated using an arrangement similar to that described by Dabiri \& Gharib (2004b). Flow from a constant-head tank, delivered by a computer-controlled solenoid valve, was allowed to drive the piston forward in its cavity. A column of fluid of was impulsively ejected from the sharp-edged nozzle by the motion of the piston and rolled up into a vortex ring which propagated through the surrounding fluid owing to its self-induced velocity.

The resulting velocity field was mapped using two-component, planar DPIV (Adrian 1991; Willert \& Gharib 1991). A pulsed Nd:YAG laser sheet was used to illuminate a plane through the non-axisymmetric nozzle and the flow was seeded with $13 \mu \mathrm{m}$ neutrally buoyant glass spheres. Light from the incident laser sheet was scattered by the glass spheres onto a CCD camera whose image plane was positioned parallel to the laser sheet. The test section measured approximately $18 \mathrm{~cm} \times 18 \mathrm{~cm}$, and the 


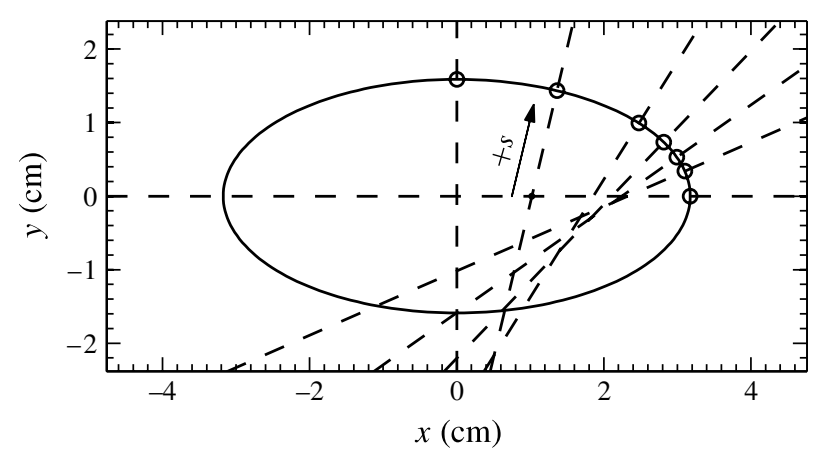

FIGURE 3. Data-collection points along the edge of the AR2 nozzle. The nozzle outline is shown in solid lines and seven data-collection points spanning the range of available curvatures are indicated by circles. The corresponding DPIV planes are denoted by the dashed lines. The arrow shows the direction of the $s$ coordinates for one DPIV plane.

resolution of the captured images was approximately $0.18 \mathrm{~mm} \times 0.18 \mathrm{~mm}$. Images were recorded at $30 \mathrm{~Hz}$, and sequential image pairs were interrogated using crosscorrelation. An interrogation window size of $32 \times 32$ pixels with a $50 \%$ overlap was utilized, yielding $63 \times 63$ velocity vectors. The velocity and vorticity fields were calculated using an in-house code, and the spatial resolution for both was approximately $3 \mathrm{~mm} \times 3 \mathrm{~mm}$. The measurement uncertainty associated with the procession technique employed was $1 \%$ for the velocity fields, and $3 \%$ for the vorticity fields (Krueger et al. 2006).

Planar DPIV data was recorded on at least two planes through each nozzle. To investigate the effect of curvature on the vortex formation number, we recorded the velocity field at seven locations between the major and minor axes of the AR2 elliptical nozzle. Figure 3 shows the outline of the AR2 nozzle, as well as the location of the seven data-collection points along the nozzle edge. The local curvature of the nozzle contour at the data planes spanned the entire available range from $\kappa=0.16 \mathrm{~cm}^{-1}$ at the minor axis to $\kappa=1.26 \mathrm{~cm}^{-1}$ at the major axis.

At each curvature point, the vortex generator was oriented such that the laser sheet was locally normal to the nozzle edge, so as to minimize out-of-plane motions. The precise alignment of the laser sheet and nozzle at each curvature point was achieved by constructing a device that secured the vortex generator at a set of predefined angles. The mounting device consisted of a three-sided box, with slots etched into the inside of the two opposing faces of the box (the sidewalls). The box sat on the bottom of the tank on its bottom plate, and acrylic plates could be fitted into the two sets of slots to complete the front and back walls of the open-top box (see figure 4). The acrylic plates were laser-cut to hold the rectangular portion of the vortex generator at a specific angle, and could be interchanged to achieve each of the preset angles precisely. The laser sheet and camera were aligned with the mounting box at the start of the experiment and remained stationary throughout data collection. Since the main body of all three vortex generators consisted of a rectangular block with the same height and width, the design of the mounting box allowed us to conduct experiments on the remaining two nozzles.

On these two nozzles, velocity data was recorded only on the two symmetry planes of each nozzle. In the case of the AR4 elliptical nozzle, the large range of curvatures along the nozzle contour $\left(\kappa_{\max } / \kappa_{\min } \approx 60\right)$ led to the pronounced deformation of the 

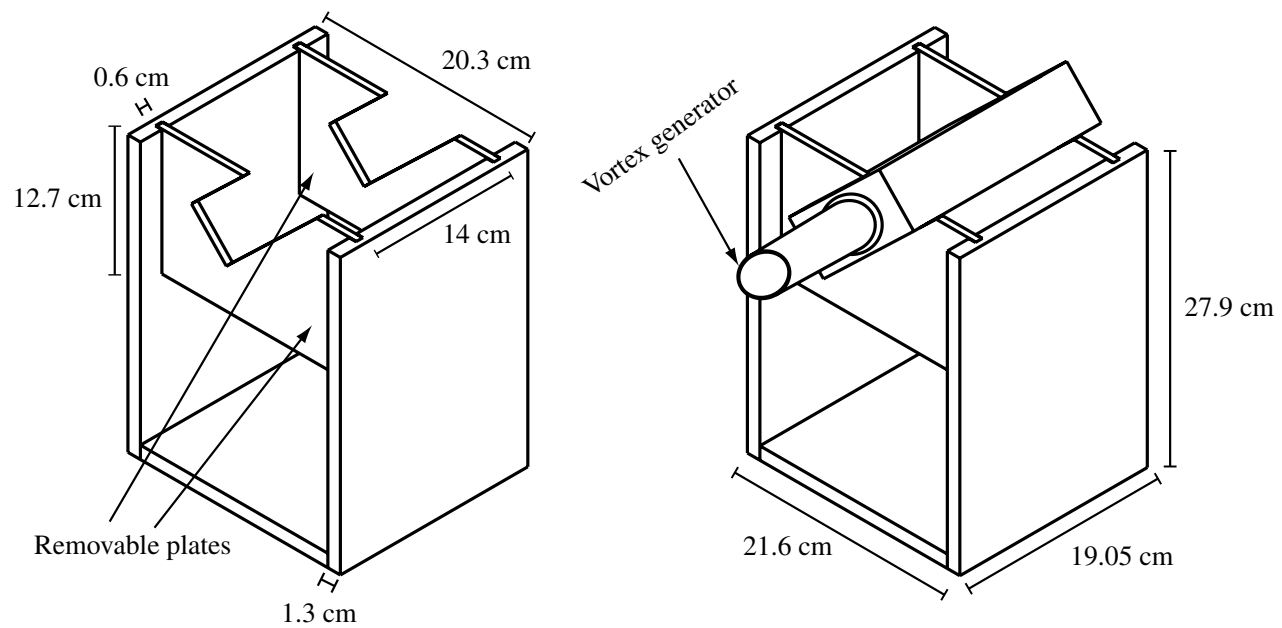

FIGURE 4. Vortex generator mounting box. Pairs of acrylic plates slid into slots on the sides of the mounting box. The plates were designed to hold the vortex generator at several predefined angles, to collect data at different curvature values and ensure proper alignment of the laser sheet and camera.

vortex ring, a process which was not adequately captured using two-component DPIV, except on the two symmetry planes of the nozzle. On the oval nozzle, the presence of four discontinuities in the local curvature along the outline of the nozzle led to highly three-dimensional motions in their vicinity, which two-component DPIV was not suited to capture on planes other than the two symmetry planes. Figure 2 shows the outline of this oval nozzle, which was constructed from two pairs of tangent circular arcs. On the two segments nearest the $x$-axis, the curvature of the nozzle was $\kappa=1.31 \mathrm{~cm}^{-1}$, whereas the curvature on the remaining two segments was $\kappa=0.33 \mathrm{~cm}^{-1}$. The four circular segments were tangent at their intersection, but there was a discontinuity in the local curvature at the four intersection points

For all three nozzle configurations, experiments were conducted at a Reynolds number based on the piston speed and equivalent diameter of the nozzle $(R e=$ $\left.\bar{U}_{p} D_{e q} / \nu\right)$ of approximately 2000, and a Reynolds number based on vortex ring circulation $\left(\Gamma_{\text {ring }} / \nu\right)$ between 3000 and 4000. The Reynolds numbers considered here are comparable with those observed in jetting swimmers such as paralarval and juvenile squid (O'Dor 1988; Bartol et al. 2009) and several species of jellyfish (Gladfelter 1973; Colin \& Costello 2002; Peng \& Dabiri 2009), which have been observed to operate at Reynolds numbers in the range of $10^{2}-10^{3}$. We did not consider the effects of varying the Reynolds number, since they have been previously characterized for circular vortex rings, and found to have little impact on the formation number, in the intermediate range of Reynolds numbers (Rosenfeld et al. 1998; Mohseni et al. 2000; Zhao et al. 2000).

The coordinate system for all experiments was defined relative to the nozzles as follows: the $z$-axis was parallel to the direction of fluid ejection, and set to coincide with the nozzle centreline, with the nozzle exit plane located at the plane $z=0$. The $x$-axis was aligned with the major axis of the nozzle and the $y$-axis coincided with the minor axis of the nozzle. An impulsive piston velocity programme was employed in all experiments and for all nozzle configurations, and an ultrasonic flow meter 


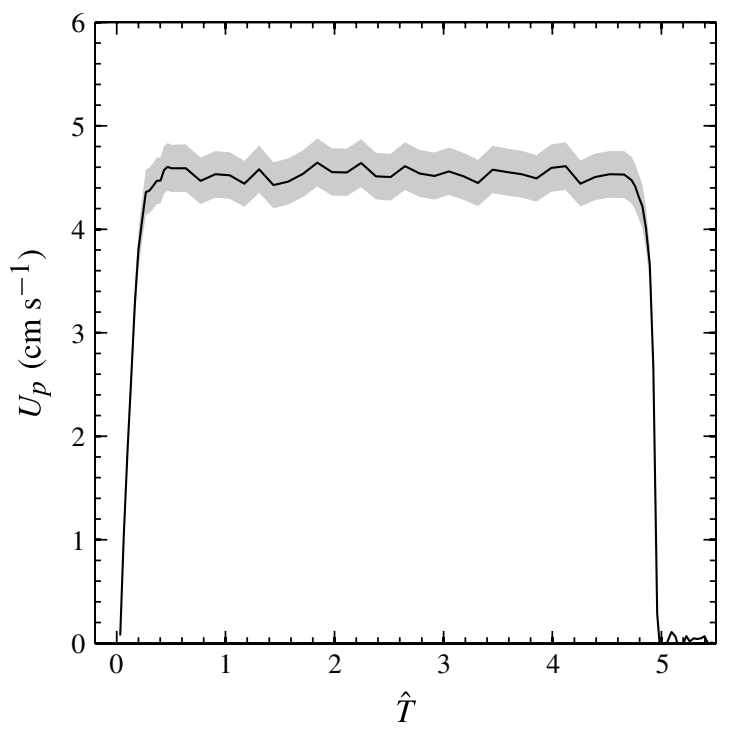

FIGURE 5. Piston velocity programme for the AR2 nozzle. The shaded region indicates the $4 \%$ measurement uncertainty band associated with the Transonic Systems T-110 ultrasonic flow meter.

was used to monitor the fluid volume displaced by the piston. Figure 5 shows a typical piston velocity programme, in this case for the AR2 nozzle. The mean piston velocity was $\bar{U}_{p}=4.5 \mathrm{~cm} \mathrm{~s}^{-1}$ for the two elliptical nozzles, and $\bar{U}_{p}=5.5 \mathrm{~cm} \mathrm{~s}^{-1}$ for the smaller oval nozzle.

For each set of experimental conditions, experiments were conducted at least three times, in order to verify the repeatability of the vortex formation process. The velocity and vorticity results presented in $\S \S 3$ and 4 are representative of those observed through repeated trials. The repeatability of the results and robustness of the vortex formation process are discussed in $\S 5$, where we consider the circulation of the non-circular vortex rings and jets.

\section{2. $L C S$}

To identify the LCS in the flow, we computed a scalar quantity known as the finitetime Lyapunov exponent (FTLE) at each point in the domain, at several time instants during vortex formation. The FTLE is a measure of the separation of neighbouring trajectories initialized near a point in the domain $\boldsymbol{x}$. The FTLE at a point $\boldsymbol{x}$ and time $t_{0}$ is given by

$$
\sigma_{t_{0}}^{T}=\frac{1}{|T|} \ln \left(\frac{\mathrm{d} \boldsymbol{x}\left(t_{0}+T\right)}{\mathrm{d} \boldsymbol{x}\left(t_{0}\right)}\right)
$$

where $\mathrm{d} \boldsymbol{x}\left(t_{0}+T\right) / \mathrm{d} \boldsymbol{x}\left(t_{0}\right)$ is the deformation tensor. Here $\sigma_{t_{0}}^{T}$ measures the maximum linearized growth rate of trajectories starting near $\boldsymbol{x}\left(t_{0}\right)$ over a time interval $T: T$ may be positive (indicating that the FTLE field is calculated by integrating trajectories in forward time) or negative (indicating integration in backward time).

The FTLE field was computed on a Cartesian grid with a resolution of $0.6 \times 0.6 \mathrm{~mm}$, using the Newman software for efficient FTLE computation (Du Toit 2010). At each time $t$, each point on the grid was advected by the flow by numerically integrating 
the velocity field data for the integration time $T$. A fourth-order Runge-Kutta scheme was used for the numerical integration, and bilinear interpolation was used whenever interpolation of the velocity data was required. The deformation tensor was then computed at each point on the grid using central differencing with neighbouring grid points. Finally, the FTLE field was computed by evaluating (2.1) at each point on the grid. Here $T$ was chosen to be $\pm 3.3 \mathrm{~s}\left(\bar{U}_{p} T / D_{e q}= \pm 4\right.$ for the elliptical nozzles and \pm 5 for the oval nozzle), since this integration time was found to yield the complete boundary of the vortex, while remaining practical in terms of computational expense.

Regions of maximum fluid particle separation (for $T>0$ ) or maximum fluid particle attraction (for $T<0$ ) produce local maximizing curves known as 'ridges' in the FTLE field (Haller \& Yuan 2000). Since LCS are analogous to the stable and unstable manifolds in time-independent systems, they can be identified from the ridges in the FTLE field. Just as two points straddling the stable manifold of a hyperbolic fixed point in a time-independent system will diverge exponentially in forward time, points straddling a repelling LCS will diverge in forward time. Likewise, points straddling an attracting LCS will diverge in backward time, a behaviour akin to the exponential divergence in backward time of points straddling the unstable manifold of a hyperbolic fixed point. Thus, repelling LCS correspond to ridges in the forward-time FTLE field, while attracting LCS correspond to ridges in the backward-time FTLE field. Like stable and unstable manifolds, LCS act as barriers to transport and as separatrices between kinematically distinct regions in the flow (Shadden et al. 2006). Hence, they are useful in identifying distinct structures in complex flows.

Although more computationally intensive than other, Eulerian, metrics the LCS method presents several advantages that are of particular relevance to the present experiment. Although streamlines have been successfully employed to determine the extent of the vortex bubble in previous studies of isolated vortex rings (Dabiri \& Gharib 2004b; Gan, Dawson \& Nickels 2012), these methods required the streamlines be computed in the frame of reference translating with the vortex ring. In the current study, long stroke ratios and non-axisymmetry were both expected to lead to the appearance of complex vortex structures translating with different and highly timedependent speeds. Hence, a Galilean transformation to a suitable frame of reference could not be easily performed, and the aforementioned vortex bubble methods were not well suited to these complex flows (O'Farrell \& Dabiri 2010). In addition, the LCS method has the advantage of being robust to errors in the velocity field, including those arising from interpolation of measured velocity data (Haller 2002), thus allowing the calculation of FTLE fields of much higher resolution than the original data (Green et al. 2007).

\section{Eulerian analysis}

\subsection{AR2 nozzle}

We recorded the velocity field at seven locations along the edge of the AR2 nozzle, where the local curvature was $\kappa=1.26 \mathrm{~cm}^{-1}$ (major axis), 1.04, $0.82,0.60,0.39,0.20$ and $0.16 \mathrm{~cm}^{-1}$ (minor axis). In order to investigate the separation of the leading vortex ring from its trailing shear layer, experiments were conducted at the longest piston stroke length possible $(21.6 \mathrm{~cm})$. In accordance with Hussain and Husain's finding that the equivalent diameter (the diameter of a circle with equivalent cross-sectional area) is a proper choice of length scale in elliptic jets with moderate aspect ratio (Hussain \& Husain 1989), distances were normalized by $D_{e q}$ and time by $D_{e q} / \bar{U}_{p}$. The resulting stoke ratio in experiments conducted on this nozzle was $L / D_{e q}=4.8$. 


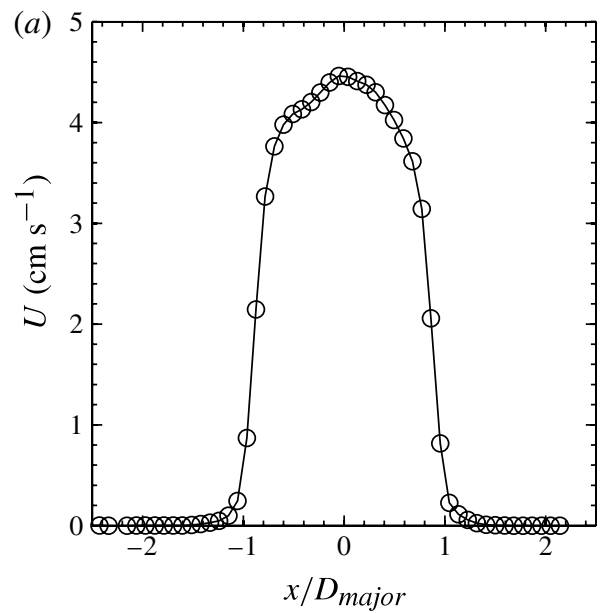

(b)

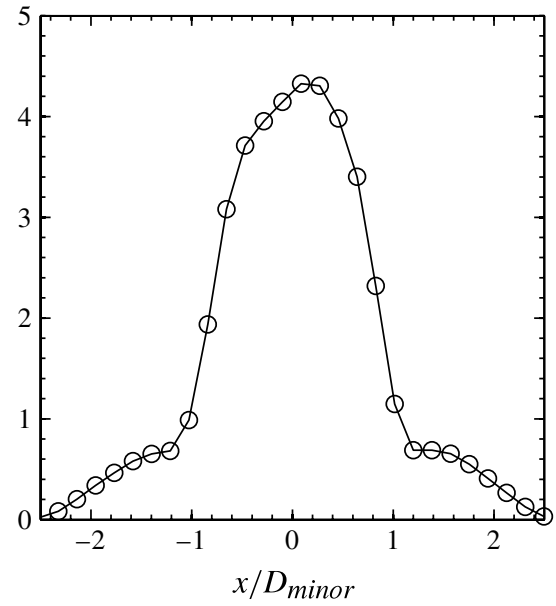

FIGURE 6. Exit velocity profile across $(a)$ the major axis plane and $(b)$ the minor axis plane of the AR2 nozzle.

Figure 6 shows typical exit velocity profiles across the major plane (figure $6 a$ ) and the minor plane (figure 6) of the AR2 nozzle, obtained from DPIV measurements. Unlike Hussain \& Husain (1989), who used a nozzle which contracted smoothly from a circular shape into an elliptical shape, and was carefully contoured to ensure a constant momentum thickness, the pistons used in this study were identical in crosssection to their respective nozzles and the nozzle was not contracting. The resulting velocity profiles at the nozzle exit were hence parabolic, as is evident in figure 6 . On the minor axis the vortex ring translated away from the nozzle exit plane at lower velocities than at the major axis (figure 7) and remained close to the nozzle during most of the fluid ejection phase. This resulted in significant induced velocities at the nozzle exit plane, which are evident from the non-zero velocities observed in the regions immediately outside of the nozzle in figure $6(b)$. The proximity of the vortex ring to the nozzle exit plane on the minor axis is also evident in the slight asymmetry of the velocity profile. At later times, the vortex ring was often found to translate slightly off the centre axis of the nozzle, resulting in an asymmetric induced velocity field. On the minor axis plane, where the vortex ring remained close to the exit plane until fluid ejection was almost complete, this asymmetry is visible in the exit velocity profile. The velocity profiles shown in figure 6 are representative of the results observed in the symmetry planes of the three nozzle configurations.

Figure 7 shows contours of the out-of-plane vorticity $\omega$ at two time instants, for all seven curvature planes. Since the flow is symmetric about the major and minor planes of the nozzle, on these two planes we recorded nearly symmetric data corresponding to the two locations where the DPIV plane intersected the nozzle contour at right angles. The major axis symmetry plane is aligned with the $x-z$ plane (figure $7 a, h$ ) in our coordinate system, and the minor axis symmetry plane is aligned with the $y-z$ plane (figure $7 g, n$ ).

On the remaining planes, the flow was not symmetric, and the DPIV plane only intersected the nozzle contour at right angles at one location. In parts $(b-f)$ and $(i-m)$ of figure 7 , results have been plotted in $s-z$ coordinates, where $s$ is the coordinate along the DPIV plane, in the direction normal to the exit jet (the $z$-axis). The origin 


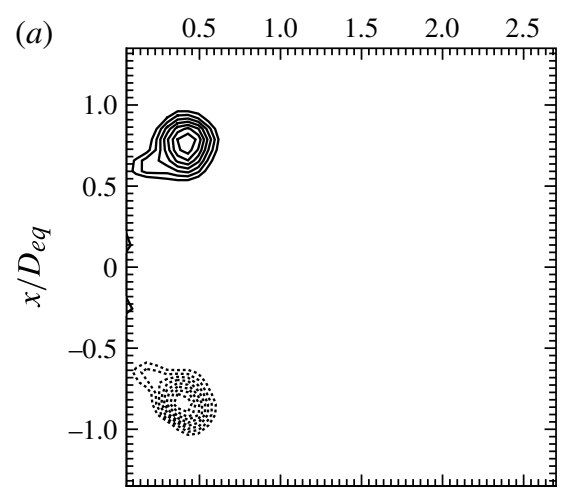

(h)
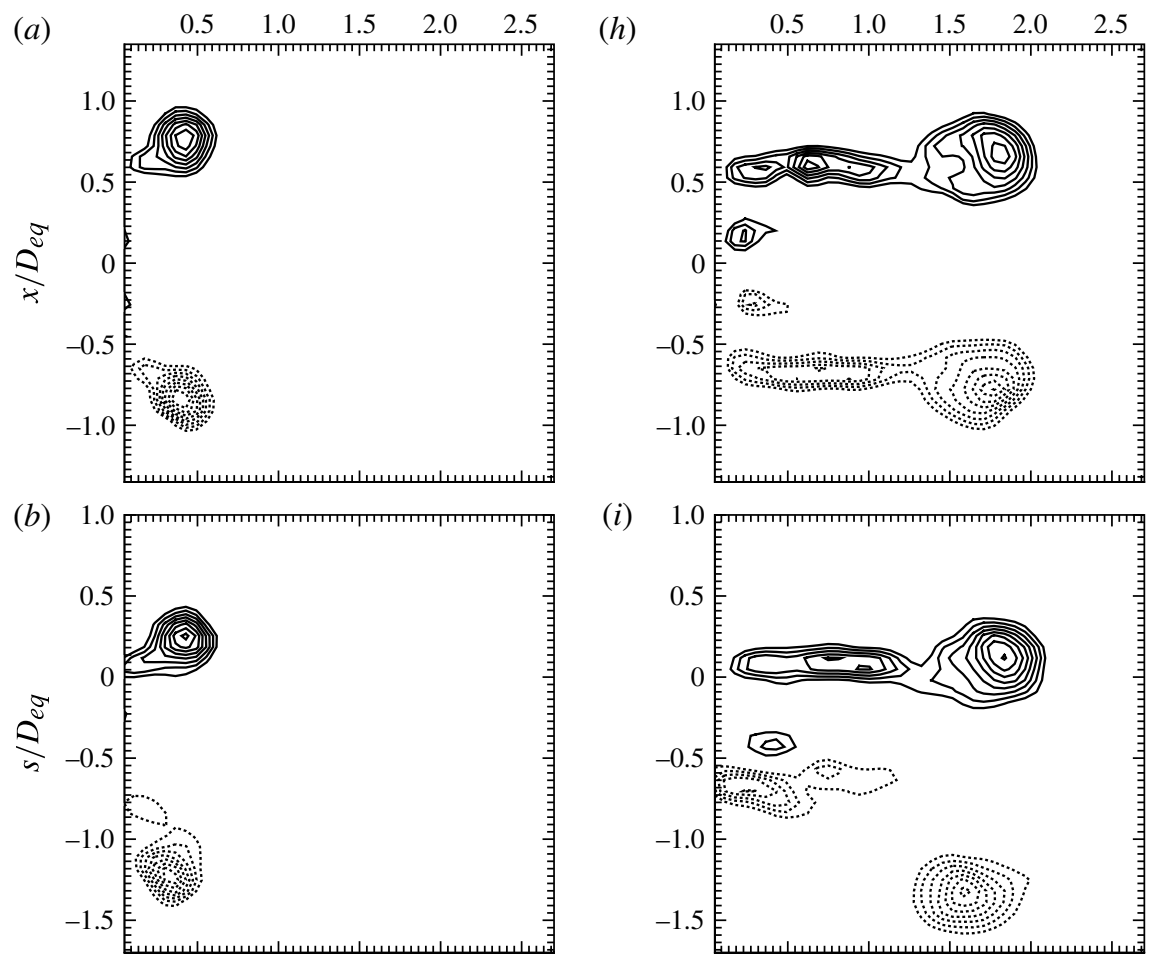

(i)
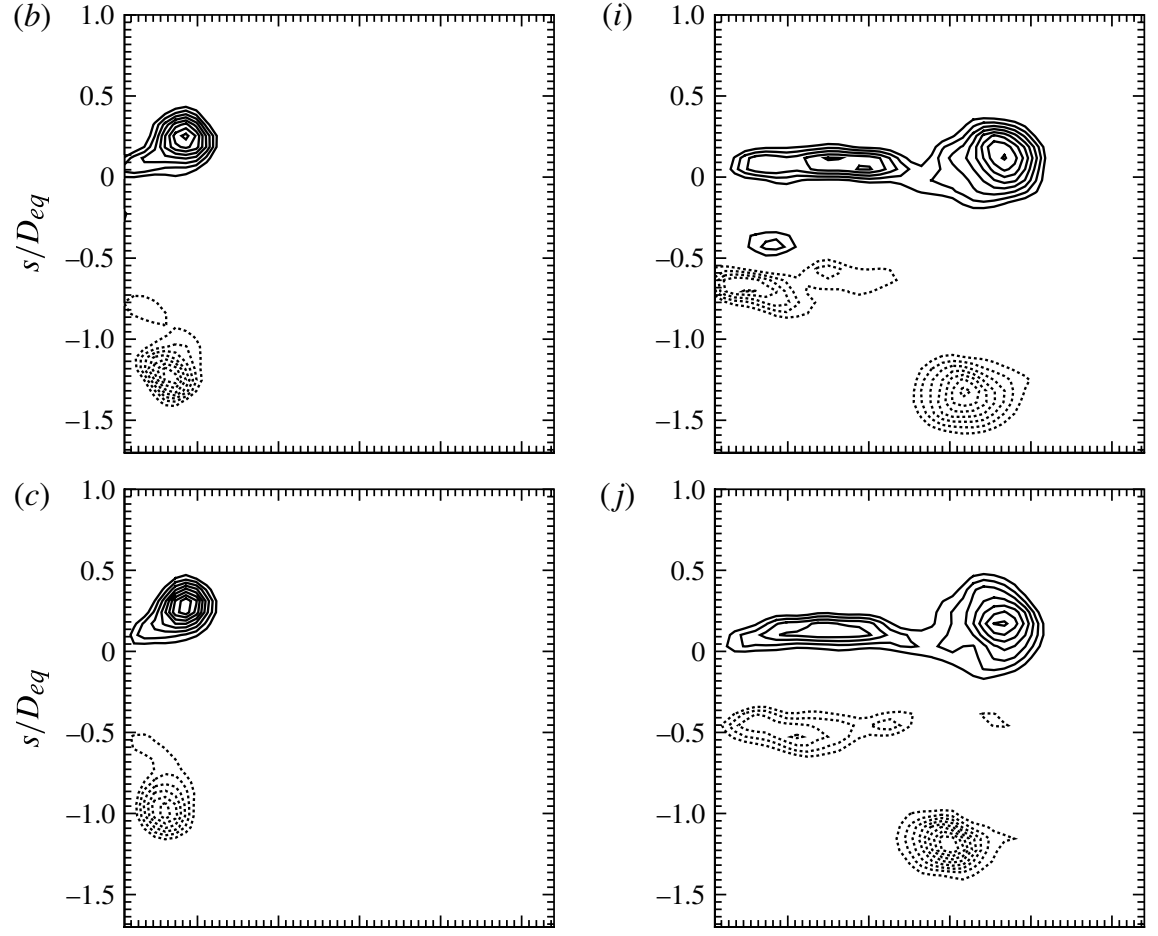

(j)
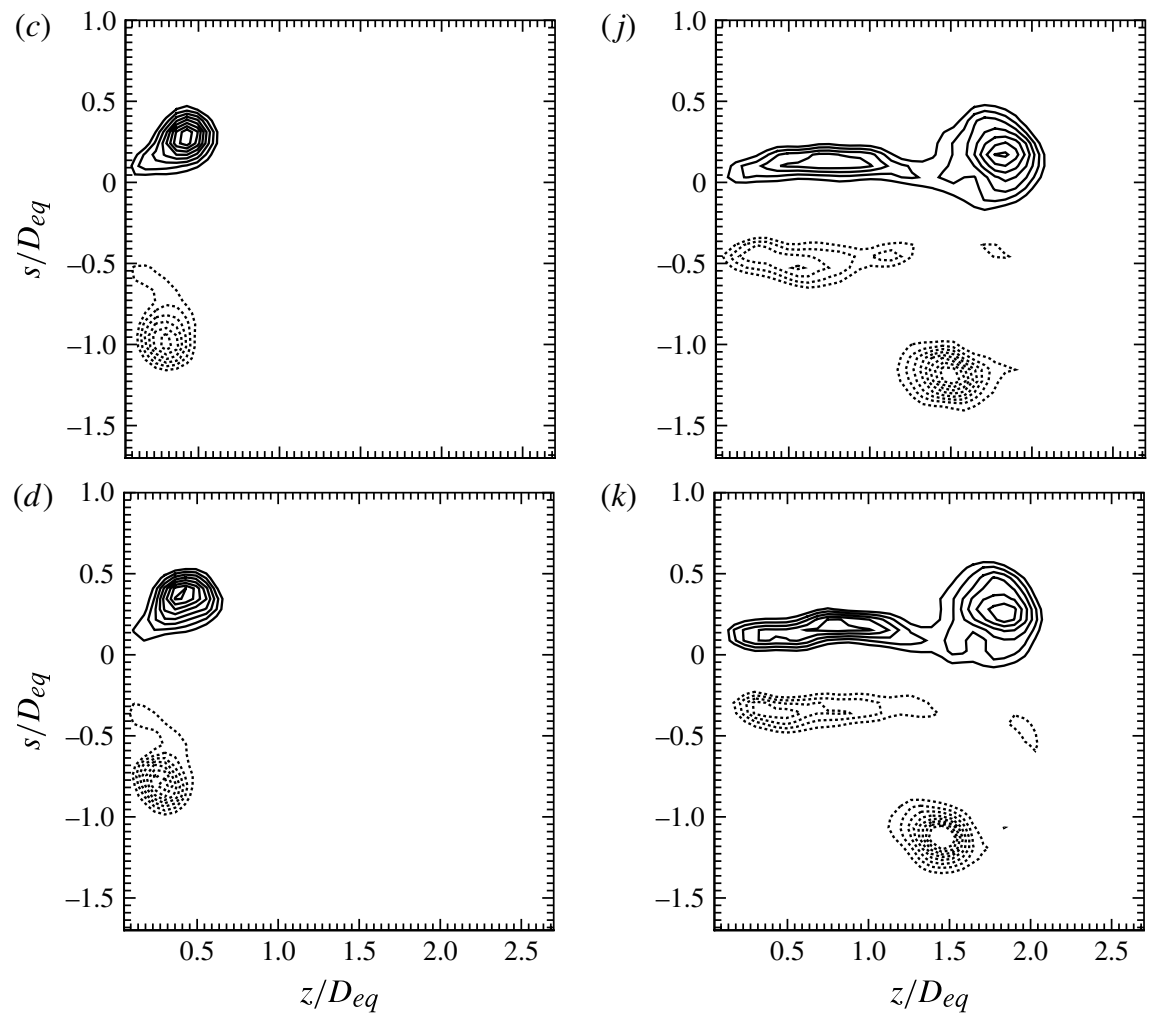

FIGURE 7. For the caption see the next page. 


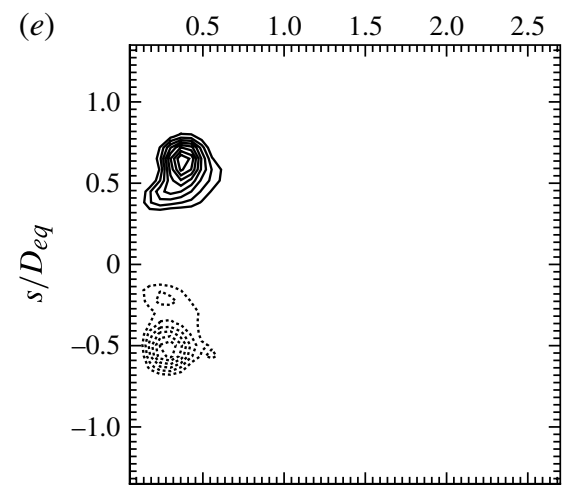

(l)
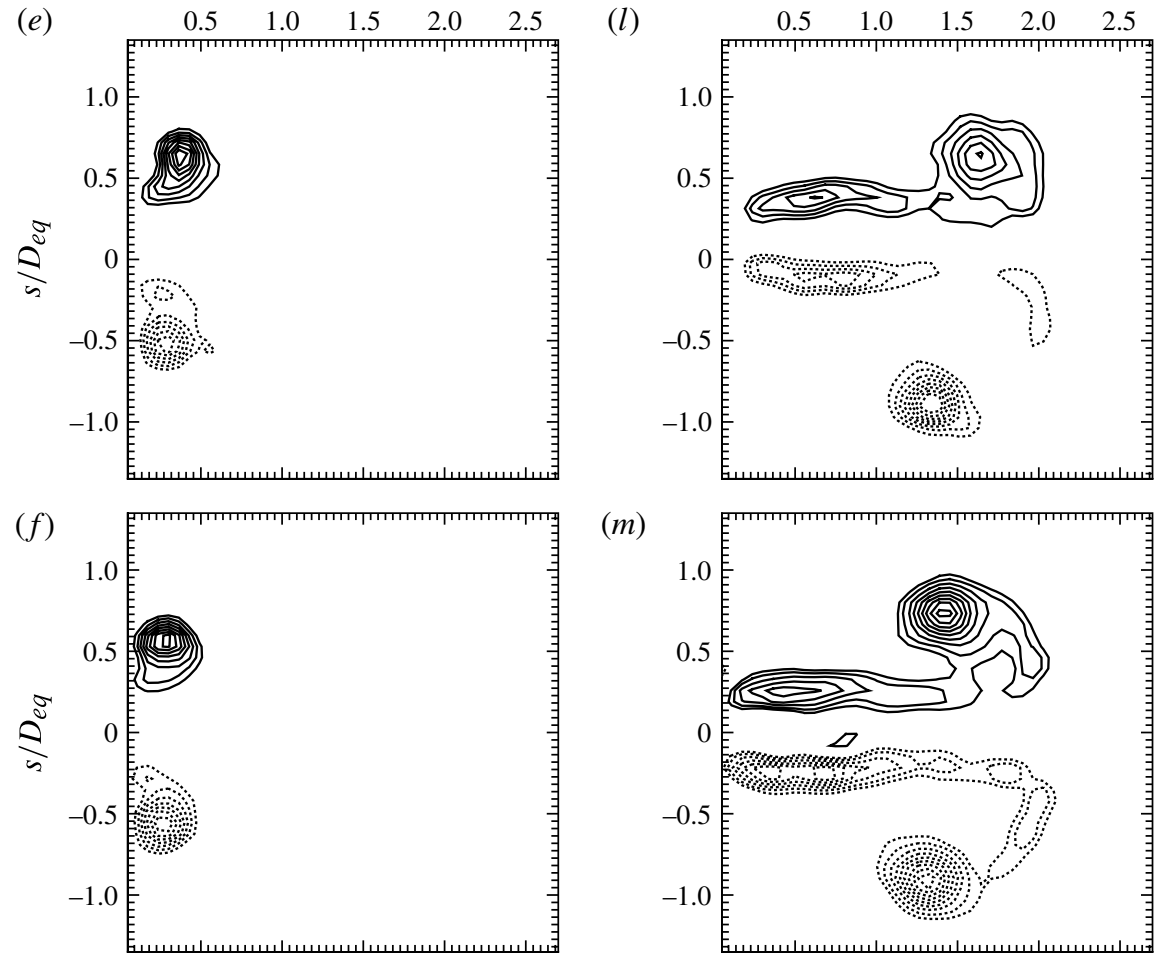

$(m)$
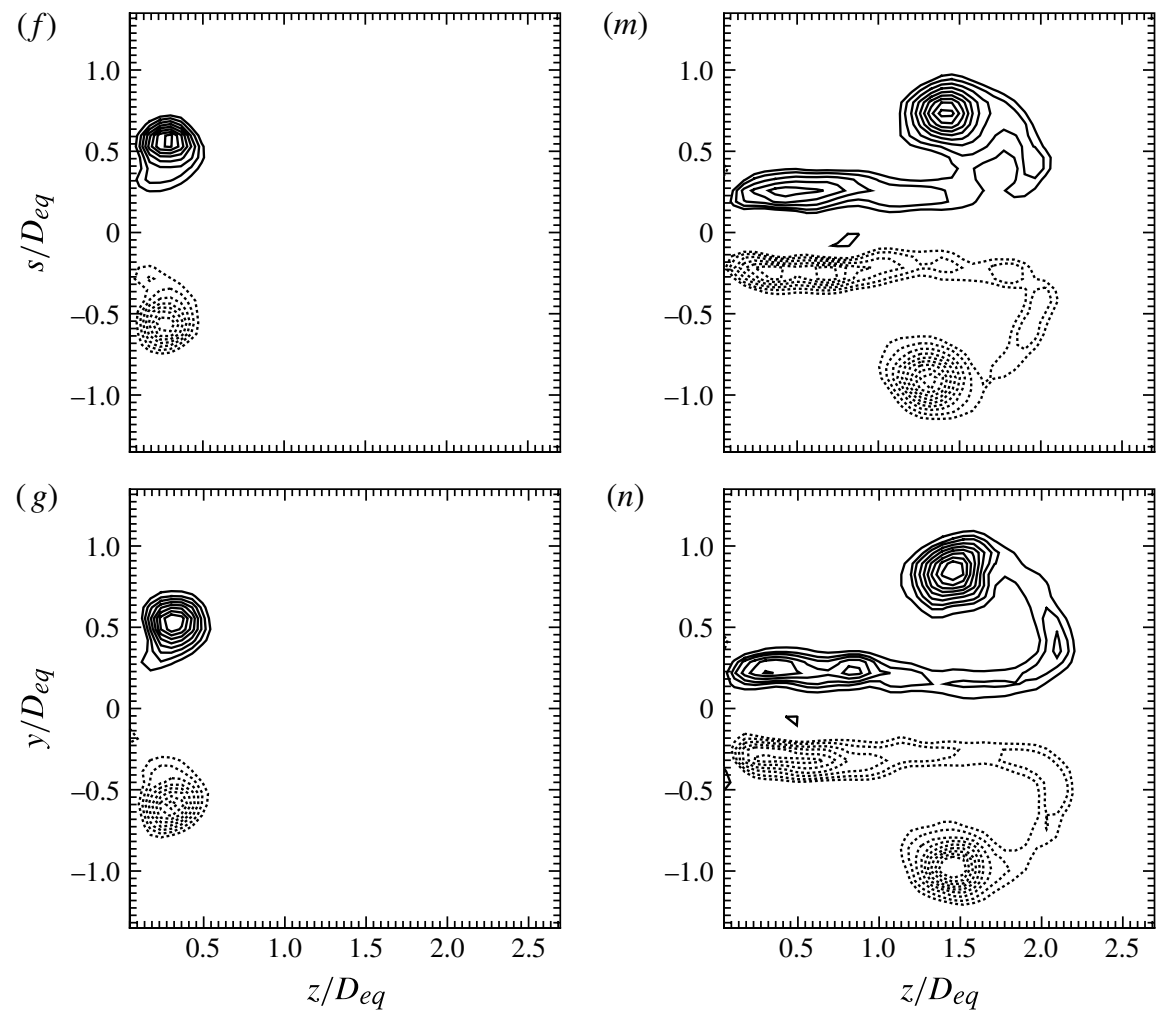

(n)

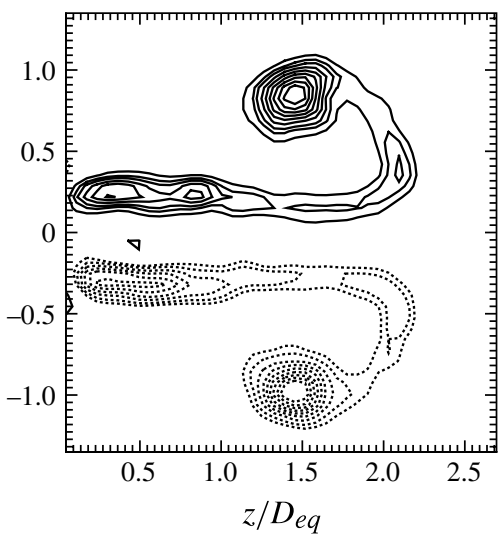

FIGURE 7. (cntd) $(a-g)$ Contours of vorticity at $\hat{T}=1.6$ on the planes of the AR2 nozzle where: (a) $\kappa=1.26 \mathrm{~cm}^{-1}$ (major axis); (b) $\kappa=1.04 \mathrm{~cm}^{-1} ;(c) \kappa=0.82 \mathrm{~cm}^{-1}$; (d) $\kappa=0.60 \mathrm{~cm}^{-1} ;(e) \kappa=0.39 \mathrm{~cm}^{-1} ;(f) \kappa=0.20 \mathrm{~cm}^{-1}$; and $(g) \kappa=0.16 \mathrm{~cm}^{-1}$ (minor axis). $(h-n)$ Contours of vorticity at $\hat{T}=4.8$ on the planes of the AR2 nozzle where: $(h)$ $\kappa=1.26 \mathrm{~cm}^{-1}$ (major axis); (i) $\kappa=1.04 \mathrm{~cm}^{-1} ;(j) \kappa=0.82 \mathrm{~cm}^{-1} ;(k) \kappa=0.60 \mathrm{~cm}^{-1} ;(l)$ $\kappa=0.39 \mathrm{~cm}^{-1} ;(m) \kappa=0.20 \mathrm{~cm}^{-1}$; and $(n) \kappa=0.16 \mathrm{~cm}^{-1}$ (minor axis). Contour levels: $\min =25 \%$ of $\omega_{\max }, \max =95 \%$ of $\omega_{\max }$, increment $=10 \%$. Dashed lines indicate negative vorticity. Flow is from left to right. 
of the $s$-axis is located at the intersection of the DPIV plane and the major axis of the nozzle (see figure 3). Only the vorticity fields in the region where $s>0$ were analysed, since it was in these regions that the DPIV plane was correctly aligned with the nozzle.

Figure $7(a-g)$ depicts the growing elliptical vortex ring in its early stages of development, at $\hat{T}=\bar{U}_{p} t / D_{e q}=1.6$. At this stage, the roll-up of the shear layer was locally two-dimensional, on a plane normal to the nozzle edge (Domenichini 2011). As a result, the vortex core cross-section was roughly the same shape and size on all data planes, and had advected approximately $0.3 D_{e q}$ downstream in all cases.

At later stages in the development of the elliptical vortex (figure $7 h-n$ ), however, significant differences were evident in the major and minor axes cross-sections. Since the velocity induced by a vortex ring is a function of the local curvature, the selfinduced velocity of the elliptical vortex ring was greater at the major axis than at the minor axis. As a result, the vortex ring deformed. On the major axis, the vortex cores advected downstream faster than anywhere else on the nozzle contour and the resulting deformation caused the cores to move towards each other along the direction parallel to the $x$-axis (figure $7 h$ ). In contrast, on the minor axis, the downstream velocity of the vortex cores reached a minimum value which was lower than the jet velocity, causing the shear layer to curve around the front of the ring in order to feed into the vortex cores (figure $7 n$ ). On this plane, the deformation caused the cores to move away from each other along the $y$-axis. As a result, what was originally the minor axis became the major axis of the deformed vortex ring; the curvature trend was reversed, and the deformation process continued.

This deformation has been observed in both isolated elliptical vortex rings (Arms \& Hama 1965; Dhanak \& de Bernardinis 1981) and elliptic jets (Hussain \& Husain 1989). In addition, the vorticity fields on the major and minor axes were qualitatively in good agreement with those reported by Adhikari (2009), who showed the evolution of the vorticity field only for stroke ratios of two and six. A stroke ratio of two resulted in the formation of only an isolated vortex ring, but at a stroke ratio of six, the geometry of the vortex ring and shear layer reported by Adhikari (2009) is qualitatively very similar to that shown in figure 7 .

The shape of the entire vortex ring can be appreciated by reconstructing the threedimensional vorticity field from the planar vorticity data. In each of the seven data planes, the out-of-plane vorticity was assumed to dominate and was taken as an approximate measure of the total vorticity magnitude. Because the deformation of the vortex ring was symmetric about the $x$ - and $y$-axes, we were able to reconstruct the deformed vortex ring from the approximate vorticity magnitude on the seven planes of available data. At each $z$-location, we interpolated the vorticity field onto a rectangular $x-y$ grid from the data available along the seven data planes. This was achieved by finding the Delaunay triangulation of the available data points, using it to construct a surface of the form $\omega=f(x, y)$, and finally interpolating this surface at the points specified by our rectangular grid. By compiling the planar data at each $z$-location into a volume, we then obtained an approximate three-dimensional field of the magnitude of the vorticity.

Figure 8 shows isosurfaces of constant vorticity magnitude for the reconstructed vorticity field at four time instants. Initially, the vortex shape closely resembled the nozzle shape, and the plane of the vortex ring was parallel to the nozzle exit plane, as shown in figure $8(a)$. However, at later stages (figure $8 c, d$ ) the vortex had deformed significantly, and the deformed vortex resembled the seam on a tennis ball 
(a)

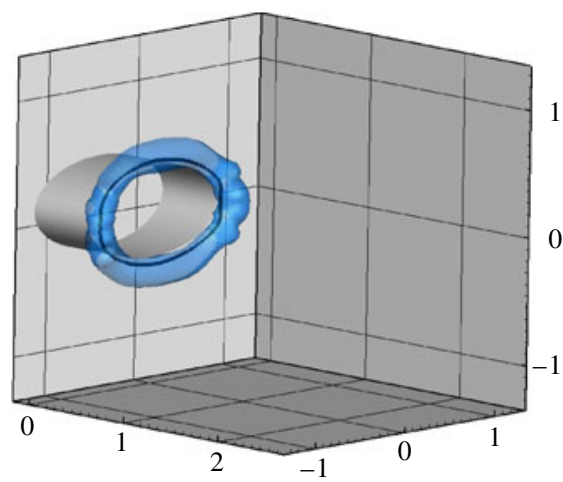

(c)

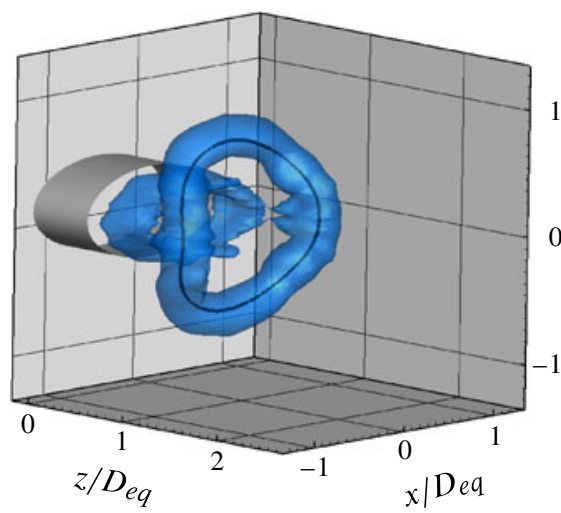

(b)

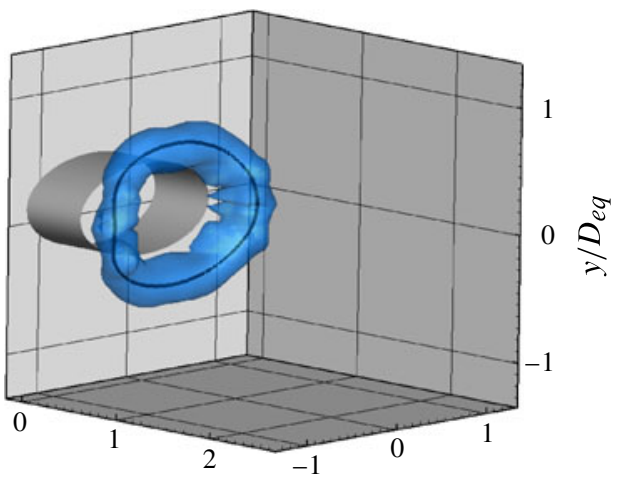

(d)

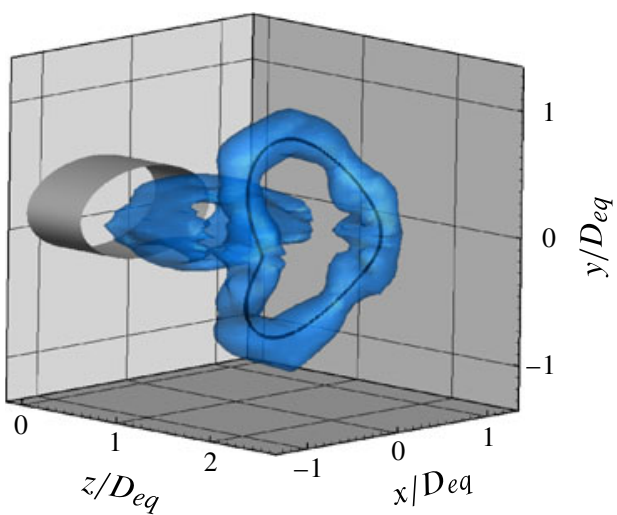

FIGURE 8 . Isosurfaces of $33 \%$ of the maximum vorticity at $(a) \hat{T}=0.5,(b) \hat{T}=1.6,(c)$ $\hat{T}=3.2$ and $(d) \hat{T}=4.8$. The AR2 elliptical nozzle is shown in grey and the black line is a spline fit through the core centre at each plane.

(Hussain \& Husain 1989). The shape of the vortex ring obtained from isosurfaces of the reconstructed vorticity was in excellent agreement with the dye visualizations of Adhikari (2009) for similar stroke ratios. As a consequence of the deformation observed in figure 8, the DPIV planes which were not aligned with symmetry axes of the nozzle did not intersect the vortex ring cores locally normally at longer times. This deformation was more significant on the AR4 and oval nozzles, and restricted our ability to record data on the off-symmetry planes, as discussed in $\S 3.2$.

In addition to deformation of the vortex ring, the development of a trailing shear layer of significant length was observed on all planes by the termination of fluid ejection at $\hat{T}=4.8$. In figure $7(n)$, it is also evident that the velocity field induced by the deforming vortex ring led to the curving of the shear layer towards the centreline of the nozzle. The motion of the shear layer towards the jet centreline led to a local decrease in its strength, and eventually to its disappearance due to vorticity cancellation, which was found to begin at $\hat{T}=6.7$. A similar phenomenon was observed for axisymmetric starting jets by Mohseni et al. (2000), and was associated with pinch-off of the leading vortex ring in that case. In contrast, no appreciable motion of the shear layer towards the $y$-axis is apparent in figure $7(h)$. 

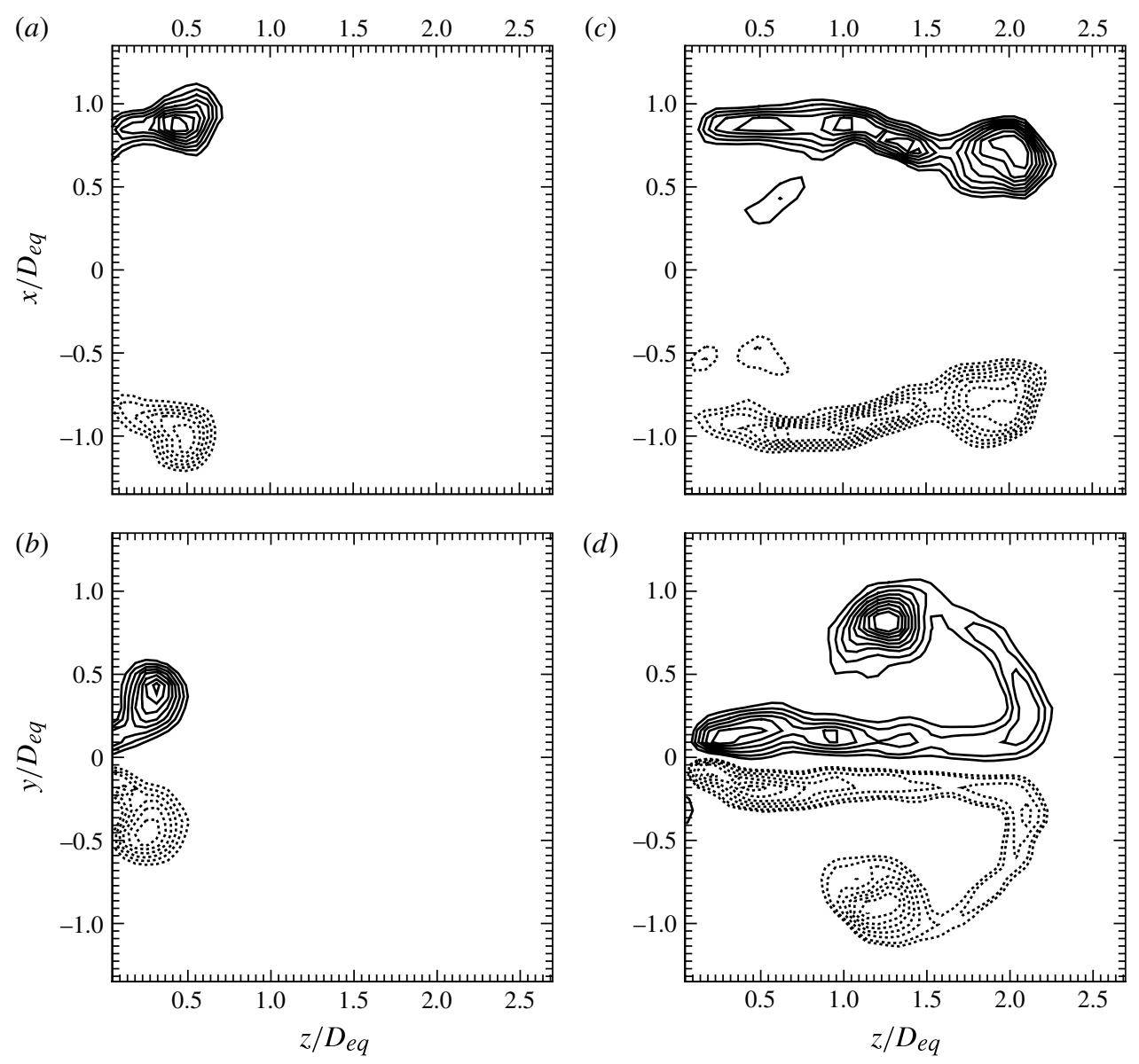

Figure 9. $(a, b)$ Contours of vorticity at $\hat{T}=1.6$, on $(a)$ the major axis and $(b)$ the minor axis of the AR4 nozzle. $(c, d)$ Contours of vorticity at $\hat{T}=4.8$, on $(c)$ the major axis and $(d)$ the minor axis of the AR4 nozzle. Contour levels: $\min =25 \%$ of $\omega_{\max }$, $\max =95 \%$ of $\omega_{\max }$, increment $=10 \%$. Flow is from left to right.

\subsection{AR4 nozzle}

A second set of experiments was conducted on the AR4 elliptical nozzle, at the largest possible stroke ratio of $L / D_{e q}=4.8$. Figure 9 shows contours of vorticity on the major and minor axes of the AR4 nozzle. As mentioned in $\S 2.1$, we were only able to collect data on planes aligned with the major and minor axes of the ellipse, since the deformation was symmetric about these two planes. On other planes along the nozzle contour, we found that the vortex deformed so rapidly as to exit the DPIV plane before the lead vortex had pinched off. The severity of the deformation is in qualitative agreement with the results of Hussain \& Husain (1989), who encountered similar effects when studying elliptic jets with aspect ratios greater than approximately 3.5 .

On the symmetry planes, however, the development of the vortex ring was qualitatively similar to that of the AR2 vortex ring shown in figure 7 , although the deformation was much more pronounced in the AR4 ring. Figure $9(a)$ and $(b)$ show the growing AR4 vortex ring in its early stages of development, at $\hat{T}=1.6$. Even at 
this early stage, the deformation of the vortex ring was already evident, since the cores had translated approximately $0.5 D_{e q}$ on the major axis, but only $0.25 D_{e q}$ on the minor axis. At $\hat{T}=4.8$, the displacement of the vortex cores towards the centreline of the nozzle along the major axis (figure $9 c$ ), and away from the centreline on the minor axis (figure 9d) was apparent. Once again, a significant trailing jet was observed on both planes at $\hat{T}=4.8$, in a configuration that is qualitatively similar to that observed by Domenichini (2011). In this case, however, significant motion of the trailing jet towards the nozzle centreline is apparent on the major axis of the nozzle. On the minor axis, the relative thickness of the shear layer compared to the nozzle minor semi-axis obscured the motion of the shear layer. However, the localized disappearance of the shear layer through vorticity cancellation was evident on this plane starting at $\hat{T}=6.2$. In contrast, no such cancellation was apparent on the major axis while the vortex ring and shear layer remained within the field of view.

\subsection{Oval nozzle}

The final set of experiments was conducted on the oval nozzle. This nozzle had a smaller cross-sectional area than the elliptical nozzles $\left(D_{e q}=3.62 \mathrm{~cm}\right.$ for the former and $D_{e q}=4.45 \mathrm{~cm}$ for the latter) and was longer, which allowed us to consider stroke ratios up to $L / D_{e q}=7.8$. Figure 10 shows contours of vorticity on the major and minor axes of the two-contour nozzle, for a stroke ratio of $L / D_{e q}=6.3$. It is evident from this figure that the development of the vortex ring ejected from the oval nozzle was qualitatively similar to that of the two elliptical vortex rings considered in this study. The deformation of the vortex ring due to the curvature dependence of the ring's propagation velocity is once again evident in figure $10(c)$ and $(d)$, as is the development of a trailing jet similar to that observed by Domenichini (2011). The motion of the shear layer towards the nozzle centreline on the minor plane is also apparent in figure $10(d)$, while the localized vanishing of the shear layer on this plane was observed after $\hat{T}=8.3$.

\section{Lagrangian analysis}

The length of the trailing jet observed from vorticity contours in all three nozzles (e.g. figure $10 c, d$ ) suggested that pinch-off was likely to have occurred on at least some portions of the nozzles. Hence, we sought to determine the formation time at which pinch-off occurred. This required applying the method of Gharib et al. (1998), i.e. comparing the total circulation ejected by the vortex generator, to the lead vortex circulation. However, in some of the vorticity distributions presented in the previous section, the boundaries of the leading vortex ring were ambiguous. On the major axis (and the data planes adjacent to it, in the AR2 case), the boundaries of the leading vortex were easily identifiable, as the vorticity field consisted solely of a shear layer and circular lead vortex core (e.g. figure 10c). On the data planes closer to the minor axis, however, the vorticity field consisted of a shear layer, circular lead vortex core, and a crescent-shaped vortex segment connecting the two (e.g. figure $10 \mathrm{~d}$ ).

Adhikari (2009) also identified this crescent-shaped vortex structure, which he termed 'flow structure 1' in his visualizations of elliptic vortex rings generated with a stroke ratio of $L / D_{e q}=2$. At these small stroke ratios, Adhikari (2009) found the crescent vortex to merge with the vortex ring completely at later times. In contrast, for larger stroke ratios (e.g. $L / D_{e q}=6$ ), he found that this structure overtook and separated from the circular vortex cores on the minor axis plane, and developed into what he termed an 'arc-like structure'. However, this arc-like structure was observed 

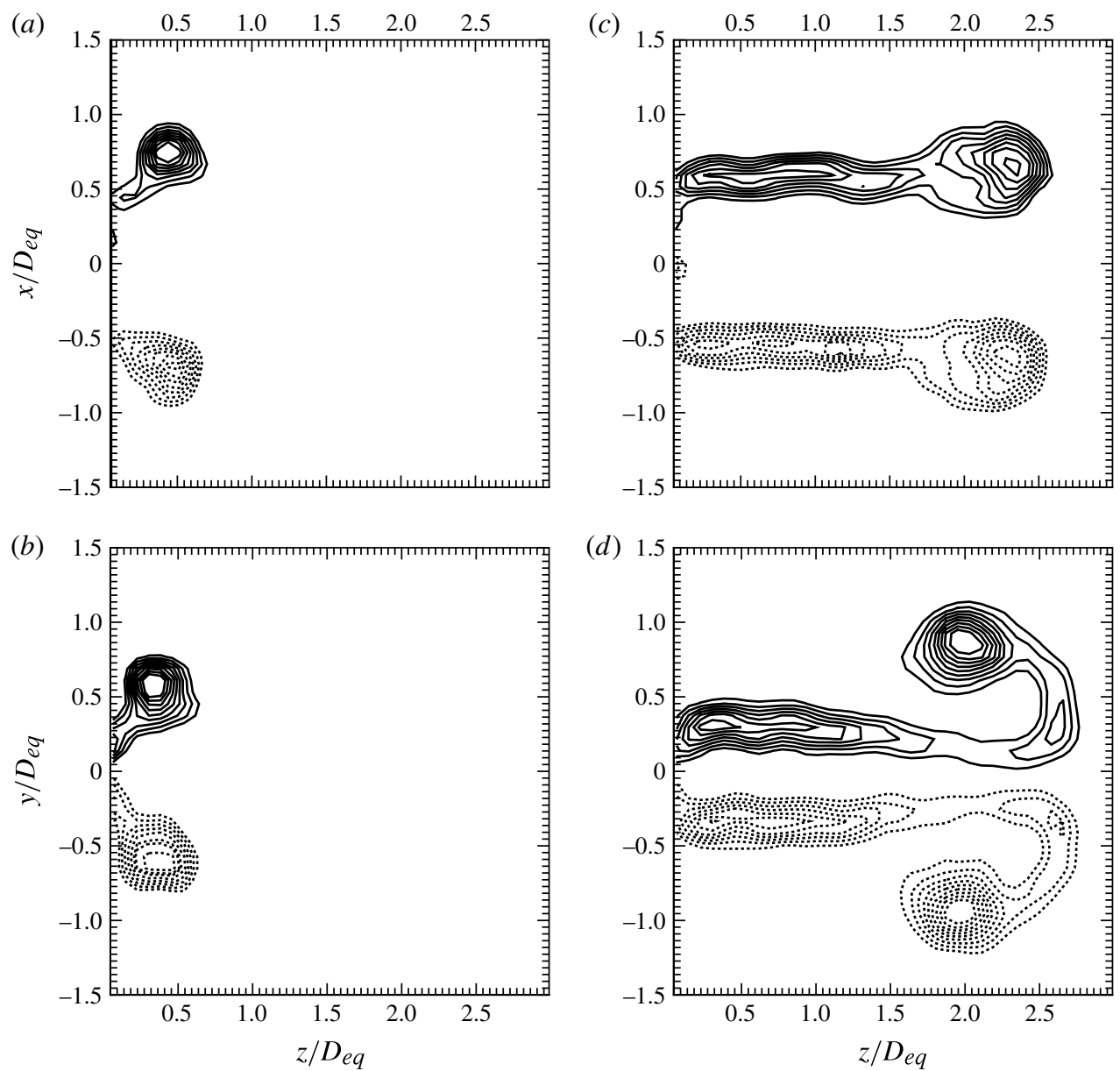

Figure 10. $(a, b)$ Contours of vorticity at $\hat{T}=1.6$, on $(a)$ the major axis and $(b)$ the minor axis of the oval nozzle. $(c, d)$ Contours of vorticity at $\hat{T}=4.8$, on $(c)$ the major axis and $(d)$ the minor axis of the oval nozzle. Contour levels: $\min =25 \%$ of $\omega_{\max }$, $\max =95 \%$ of $\omega_{\max }$, increment $=10 \%$. Flow is from left to right.

from dye visualizations to connect to the outer regions of the core on the major axis (cf. figures 4-41 and 4-42 of Adhikari (2009)). As a result, it was unclear whether the crescent vortex observed in contours of vorticity on the minor axis of all three nozzles constituted a part of the leading vortex ring or a separate flow entity.

In these situations, where the vortex structure consists of several component vortices moving at different velocities, Eulerian quantities such as vorticity can prove difficult to interpret. This is especially true when the perceived size and shape of the vortices varies depending on the thresholds or contour levels in use. In contrast, LCS often prove useful in these cases because they are frame invariant, their location is independent of threshold selection, and they identify kinematically distinct regions even in complex vortex flows (Shadden et al. 2006; Green et al. 2007; O'Farrell \& Dabiri 2010). Therefore, to resolve the ambiguity in defining the boundary of the leading vortex ring, we considered the LCS in the flow. 

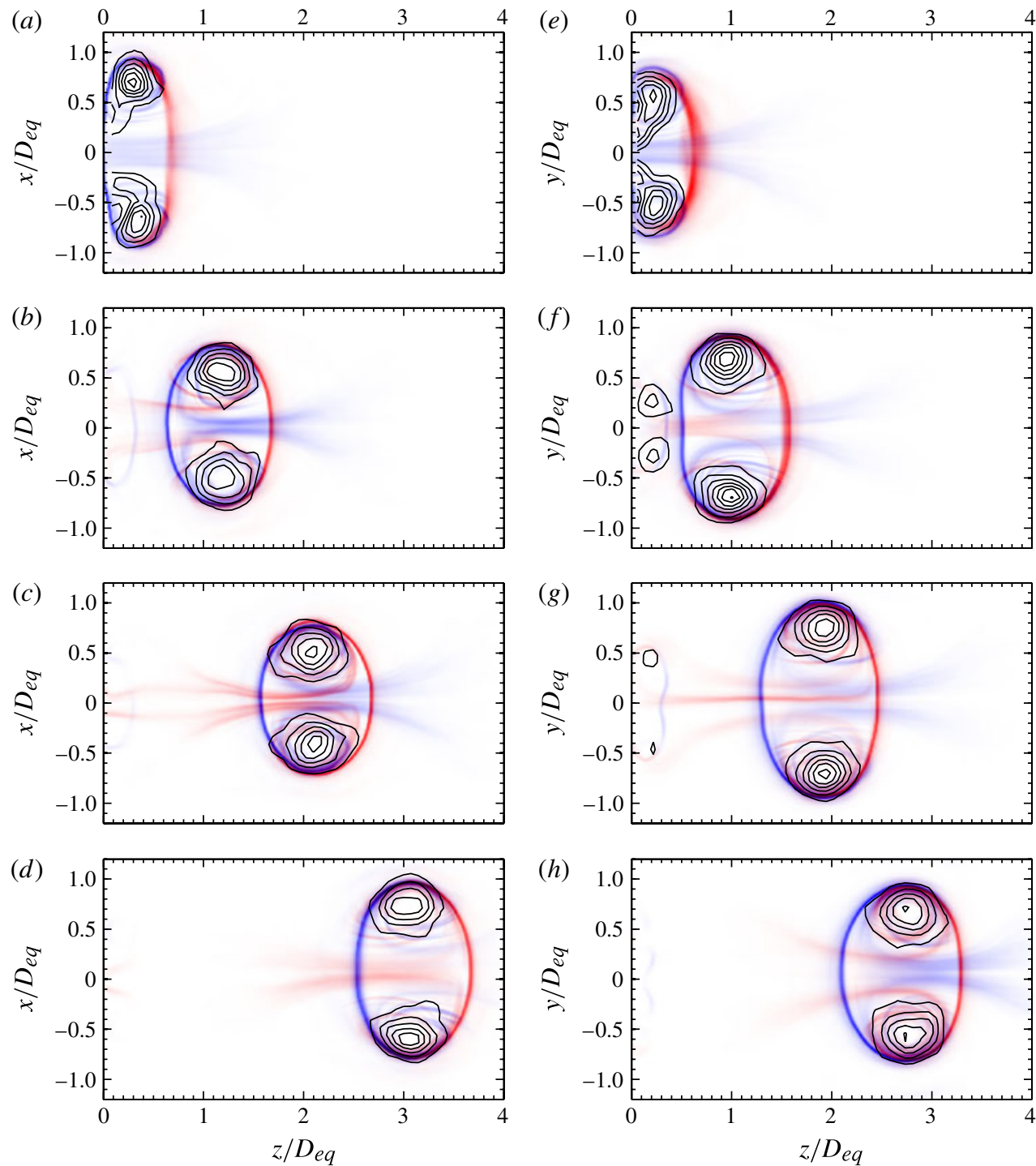

FIGURE 11. Contours of the forward-time (white to blue) and backward-time (white to red) FTLE for a vortex ring formed from the oval nozzle with a stroke ratio of 1.8: $(a-d)$ major axis; $(e-h)$ minor axis. Contours of vorticity are superimposed: $\min =10 \%$ of the maximum $\omega, \max =95 \%$ of the maximum $\omega$, increment $=15 \%$. Formation time increases down each column: $\hat{T}=1.2,4.1,7,10$.

Since the oval nozzle had a smaller cross-sectional area and a longer cavity, it allowed us to consider a greater range of stroke ratios than the elliptical nozzles. As a result, we conducted a set of experiments on the oval nozzle, at four stroke ratios ranging from 1.8 to 7.8 , and we observed the evolution of the crescent vortex. For each of these cases, we recorded the velocity and vorticity fields and computed the FTLE field at several time steps using the methods outlined in $\$ 2.2$. In figures 11-14 we present the forward-time and backward-time FTLE fields at four separate time instants, for experiments conducted using stroke ratios of $L / D_{e q}=1.8,3.3,6.3$ and 7.8. 
The forward-time and backward-time FTLE fields are represented in the form of contours ranging from white to blue and white to red, respectively, and contours of vorticity are superimposed on the FTLE fields for reference.

Figure 11 shows contours of vorticity and FTLE for an experiment conducted using a stroke ratio of 1.8. In this case, the stroke ratio was sufficiently small as to result in the formation of an isolated circular vortex core on both the major and minor axis planes of the oval nozzle. Figure 11(a,e), depict the vortex ring at $\hat{T}=1.2$, on the major and minor axes of the nozzle, respectively. At this stage, fluid ejection had not yet stopped, so the rear boundary of the vortex (formed by the repelling LCS) was not complete. By $\hat{T}=4.1$, fluid ejection had stopped and the attracting and repelling LCS formed the boundaries of the vortex ring on both planes (figure $11 b, f$ ). In this case, the LCS structure on both planes qualitatively resembled that observed by Shadden et al. (2006) for isolated circular vortex rings formed at similar stroke ratios. In addition, the time-varying deformation of the isolated vortex ring was evident from the oscillations in the diameter of the vortex ring on both the major and minor planes (figure $11 b-d$ and $f-h$ ).

When the stroke ratio was increased to 3.3 , a small crescent vortex was evident on the minor plane during formation (figure 12f,g). Pinch-off was not observed in this case, so nearly all of the vorticity ejected from the nozzle was entrained into the vortex ring and the remainder dissipated due to viscous diffusion. Once the vorticity in the short shear layer had been entrained into the leading vortex core, the LCS structure largely resembled that observed in the case where $L / D_{e q}=1.8$ (cf. figure $12 c, g$ and figure $11 b-d, f-h)$. However, two new branches were observed to form in the repelling LCS, and they are shown to intersect the crescent vortices on the minor plane at $\hat{T}=5.9$ in figure $12(\mathrm{~g})$. The small amounts of vorticity which were separated from the main vortex core by these new branches in the repelling LCS, were found to overtake the leading vortex core and form the flow structure observed by Adhikari (2009) (figure 12h). At $\hat{T}=8.6$, the core was also observed to become more diffuse on the major axis, leading to the appearance of a gap between the attracting and repelling LCS on this plane (figure 12d). This structural change in the LCS was indicative of the connection of the flow structure identified on the minor axis, to the outer regions of the core on the major axis as observed in Adhikari's dye visualizations at larger stroke ratios. Throughout the formation process, the crescent vortex was found to remain within the bounds of the leading vortex formed by the LCS, thus indicating that the crescent vortex was part of the leading vortex ring. Portions of the crescent vortex did separate from the cores in finite time and this separation was concurrent with the disintegration of the vortex core on the major axis plane.

The LCS structure observed in figure 12 for a stroke ratio of 3.3 persisted for higher stroke ratios, including those exceeding 4 , in which the formation of a persistent trailing jet was observed. Figure 13 shows the LCS structure and contours of vorticity for the experiment conducted with a stroke ratio of 6.3 , and previously analysed in §3.3. In this case, a clear crescent vortex was observed in the minor plane (figure $13 \mathrm{~g}$ ), and the portions of this crescent vortex delimited by the branching repelling LCS were observed to form a much stronger vortex pair which overtook the leading vortex core at large formation times (figure 13h). The breakup of the core on the major axis at large formation times, due to the connection of the vortex pair on the minor axis to the outer regions of the core on the major axis, was found to be more pronounced in this case as well (figure $13 d$ ). In addition, a significant amount of vorticity was found to trail in the regions behind the boundaries of the leading vortex 

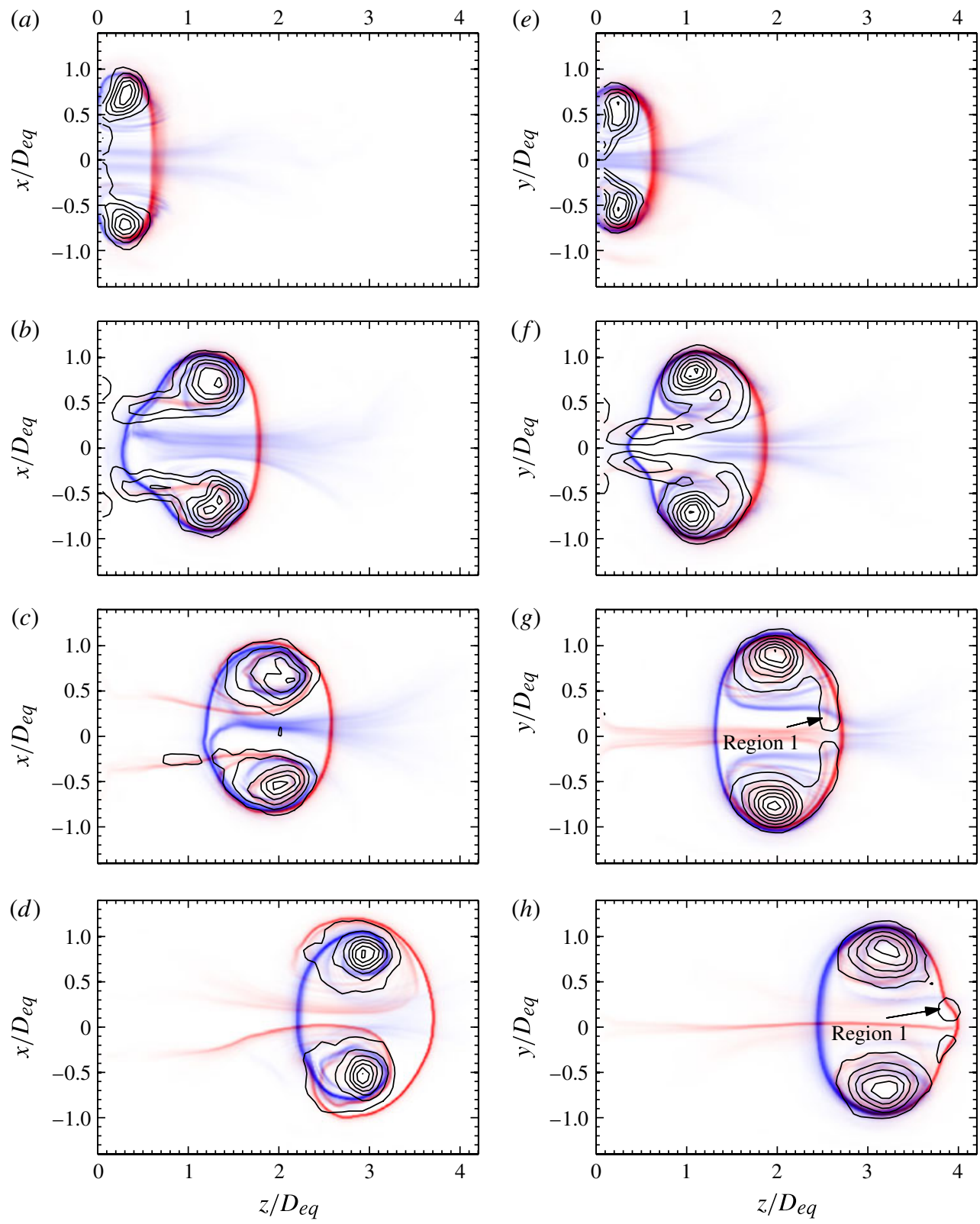

FIGURE 12. Contours of the forward-time (white to blue) and backward-time (white to red) FTLE for a vortex ring formed from the oval nozzle with a stroke ratio of 3.3: $(a-d)$ major axis; $(e-h)$ minor axis. Contours of vorticity are superimposed: $\min =10 \%$ of the maximum $\omega, \max =95 \%$ of the maximum $\omega$, increment $=15 \%$. Formation time increases down each column: $\hat{T}=1.2,4.0,5.9,8.6$.

core and a secondary vortex ring was even observed to form in the minor plane by $\hat{T}=9.1$ (figure 13h). However, unlike the secondary vortex in the wake, the crescent vortex was found to remain within the bounds formed by the LCS for the duration of the vortex formation process. 

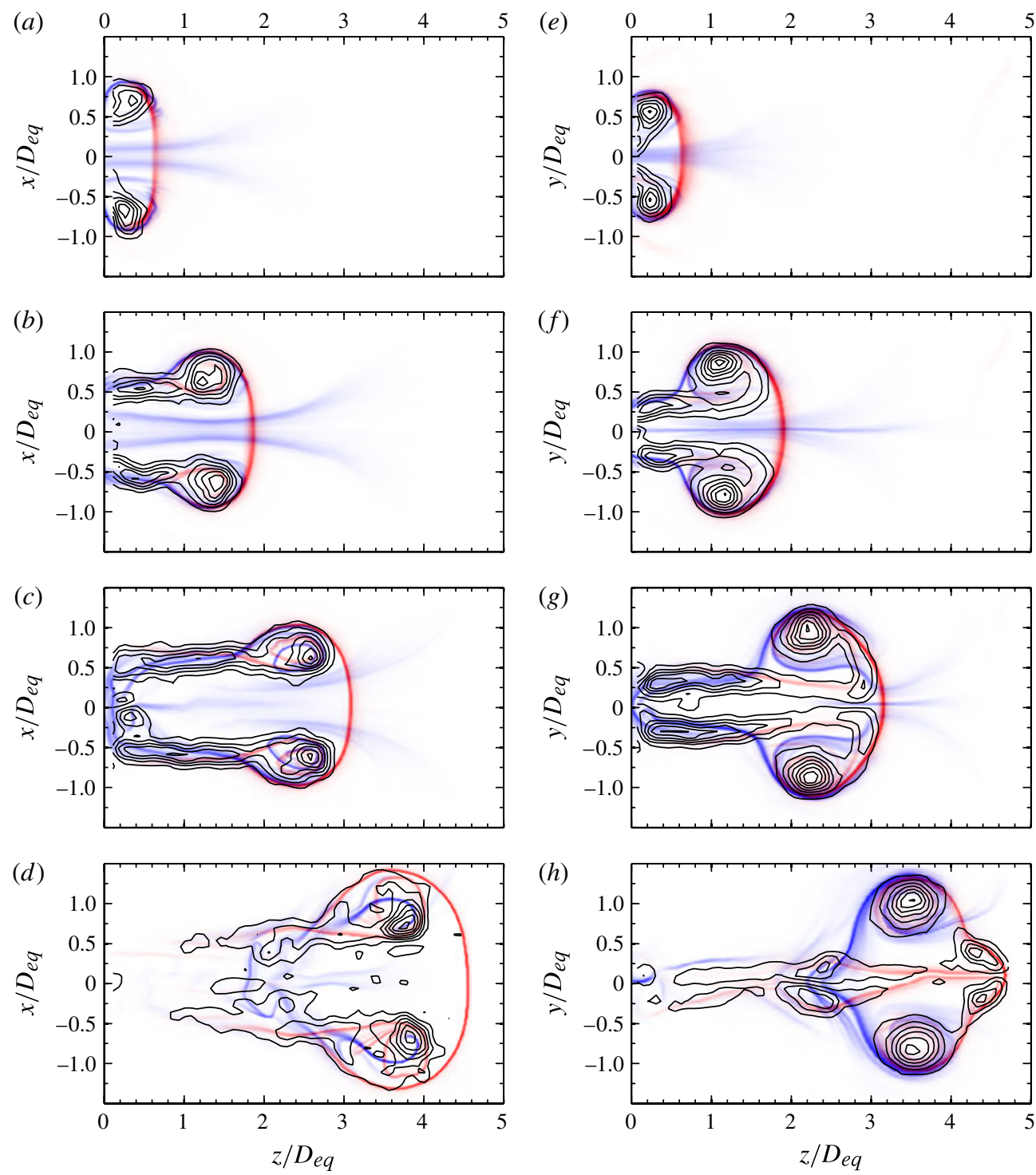

FIGURE 13. Contours of the forward-time (white to blue) and backward-time (white to red) FTLE for a vortex ring formed from the oval nozzle with a stroke ratio of 6.3: $(a-d)$ major axis; $(e-h)$ minor axis. Contours of vorticity are superimposed: $\min =10 \%$ of the maximum $\omega, \max =95 \%$ of the maximum $\omega$, increment $=15 \%$. Formation time increases down each column: $\hat{T}=1.2,4.0,6.4,9.1$.

A similar structure was observed in the experiments conducted on the oval nozzle at the maximum achievable stroke ratio of 7.8. Figure 14 shows the LCS structure and contours of vorticity for this stroke ratio. In this case, several Kelvin-Helmholtz-type vortices were seen to develop in the wake of the leading vortex at long formation times (figure 14h). With the formation of new vortices in the trailing jet, new repelling LCS were also observed to appear in the wake, and the merger of these repelling LCS due to the merger of vortices was also observed. New repelling LCS similar 

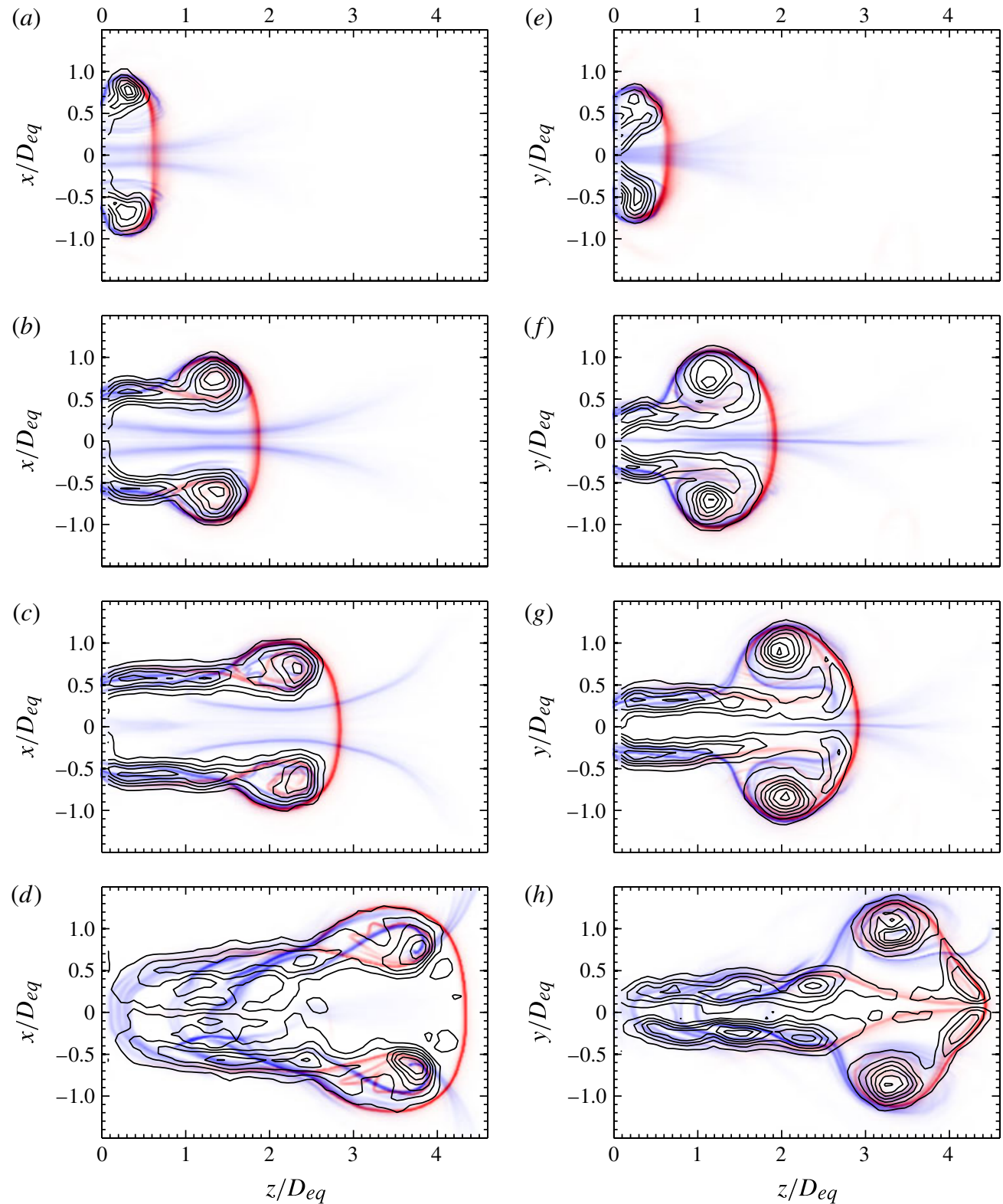

FIGURE 14. Contours of the forward-time (white to blue) and backward-time (white to red) FTLE for a vortex ring formed from the oval nozzle with a stroke ratio of 7.8: $(a-d)$ major axis; $(e-h)$ minor axis. Contours of vorticity are superimposed: $\min =10 \%$ of the maximum $\omega, \max =95 \%$ of the maximum $\omega$, increment $=15 \%$. Formation time increases down each column: $\hat{T}=1.2,4.0,5.9,8.6$.

to those observed in a circular starting jet with a long stroke ratio by O'Farrell \& Dabiri (2010), are visible in the wake in figure 14(e,h). However, the kinematics of the crescent vortex remained unchanged.

The main features of the LCS structure observed in figures 13 and 14 can also be identified in figures 15 and 16, which depict contours of vorticity superimposed on 

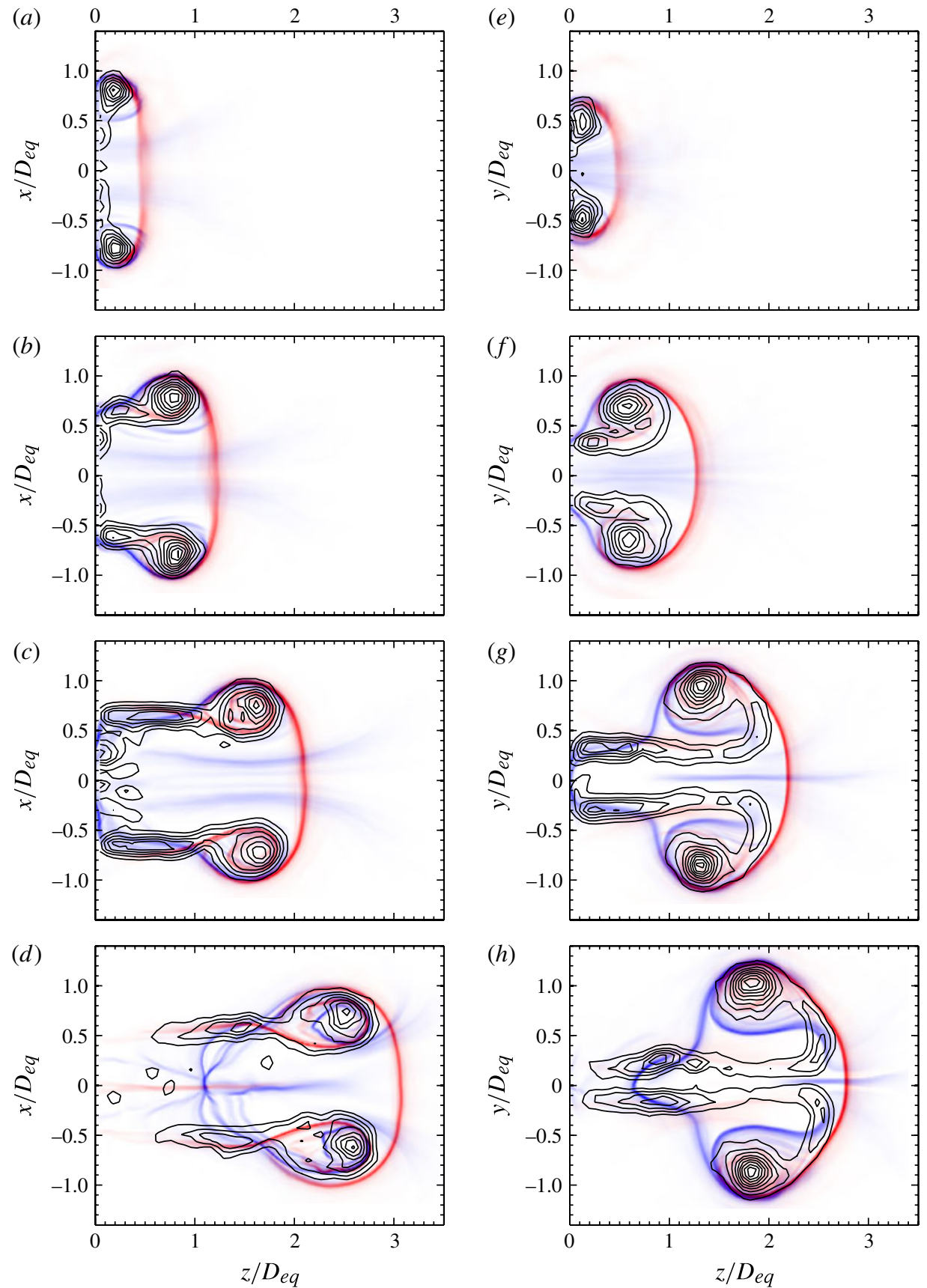

FIGURE 15. Contours of the forward-time (white to blue) and backward-time (white to red) FTLE for a vortex ring formed from the AR2 nozzle with a stroke ratio of 5.8: $(a-d)$ major axis; $(e-h)$ minor axis. Contours of vorticity are superimposed: $\min =10 \%$ of the maximum $\omega, \max =95 \%$ of the maximum $\omega$, increment $=15 \%$. Formation time increases down each column: $\hat{T}=0.8,2.7,4.7,6.7$. 

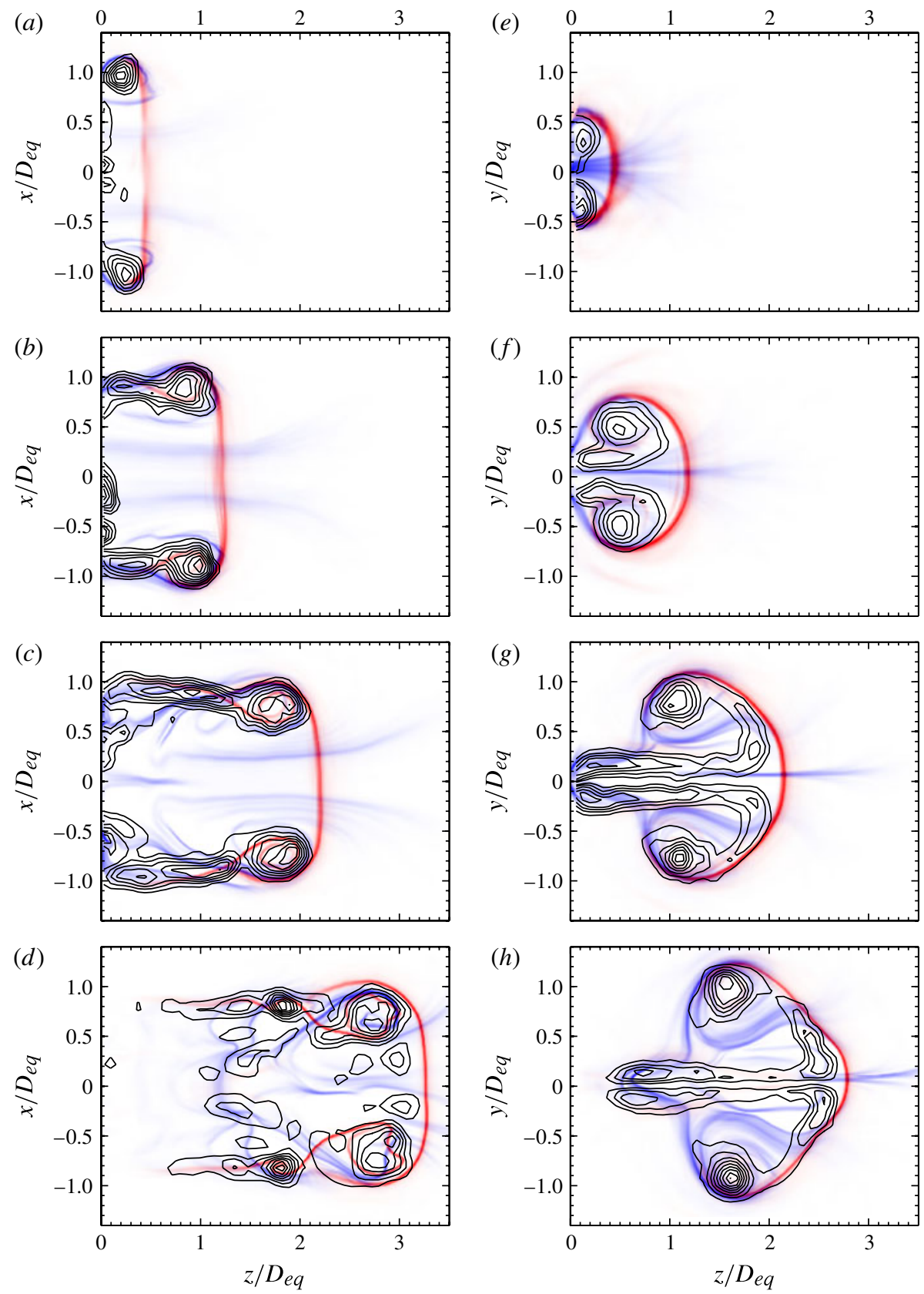

FIGURE 16. Contours of the forward-time (white to blue) and backward-time (white to red) FTLE for a vortex ring formed from the AR4 nozzle with a stroke ratio of 5.8: $(a-d)$ major axis; $(e-h)$ minor axis. Contours of vorticity are superimposed: $\min =10 \%$ of the maximum $\omega, \max =95 \%$ of the maximum $\omega$, increment $=15 \%$. Formation time increases down each column: $\hat{T}=0.9,2.8,4.8,6.8$. 


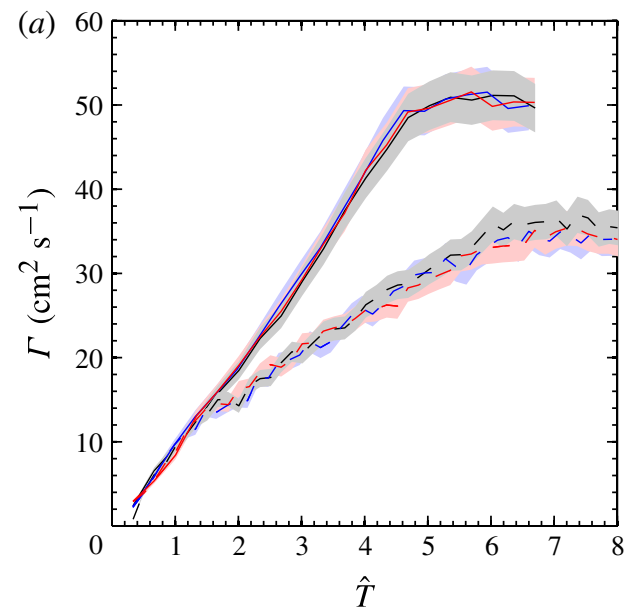

(b)

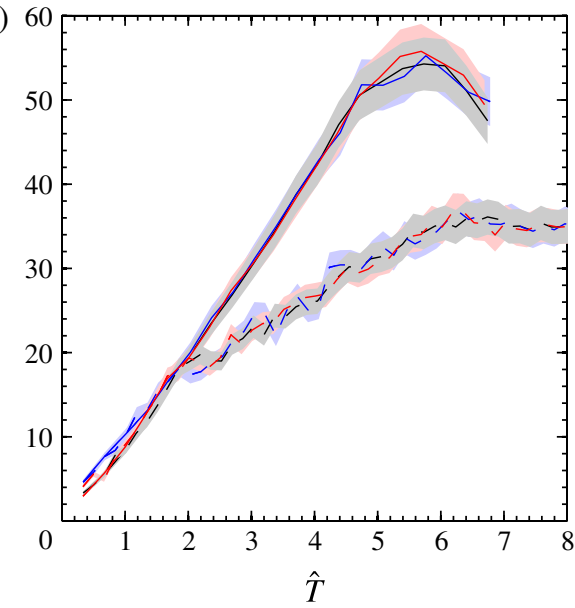

FIGURE 17. Time history of the total circulation emanating from the nozzle (solid lines) and the circulation in the lead vortex (dashed lines) on $(a)$ the major axis of the AR2 nozzle and (b) the minor axis. The different line colours denote separate trials at the same experimental conditions, with the solid and dashed lines in a particular colour corresponding to the total and vortex ring circulation measured in the same trial. The error in the measurements was $\approx 6 \%$, and it is indicated by the shaded bands.

the contours of the forward-time and backward-time FTLE fields for the AR2 and AR4 nozzles, respectively. Once again, the crescent vortex was found to remain within the boundaries of the vortex ring during formation, and branches of the repelling LCS were found to delimit the portions of the crescent vortex which would later form the arc-like structure observed by Adhikari (2009). Therefore, the LCS analysis revealed that the crescent vortex observed in the minor planes of all three nozzles was part of the leading vortex ring. In addition, the LCS predicted the development of the arc-like structure, which was found to connect portions of the crescent vortex to the outer boundaries of the core on the major axis. The deformation and eventual break-up (Adhikari 2009) that results from the formation of this arc-like structure, while interesting, is not relevant to the study of vortex pinch-off, since it occurs after the vortex has separated from the nozzle. Thus, it has no impact on the properties of the vortex ring during formation.

\section{Vortex formation number}

Following Gharib et al. (1998), we computed the total circulation emanating from the piston-cylinder apparatus, as well as the circulation in the leading vortex ring, by integrating the vorticity contained within the lowest detectable contours. Figure 17 shows the time history of the total circulation emanating from the nozzle, as well at the circulation in the leading vortex ring, for both the major plane (figure 17a) and the minor plane (figure 17b) of the AR2 nozzle. For each plane, results corresponding to three separate trials are presented, each denoted by a different line colour. In all cases, the time histories of both the total circulation and the leading vortex ring circulation were found to match very closely between trials and the vortex formation process was found to be very robust. 

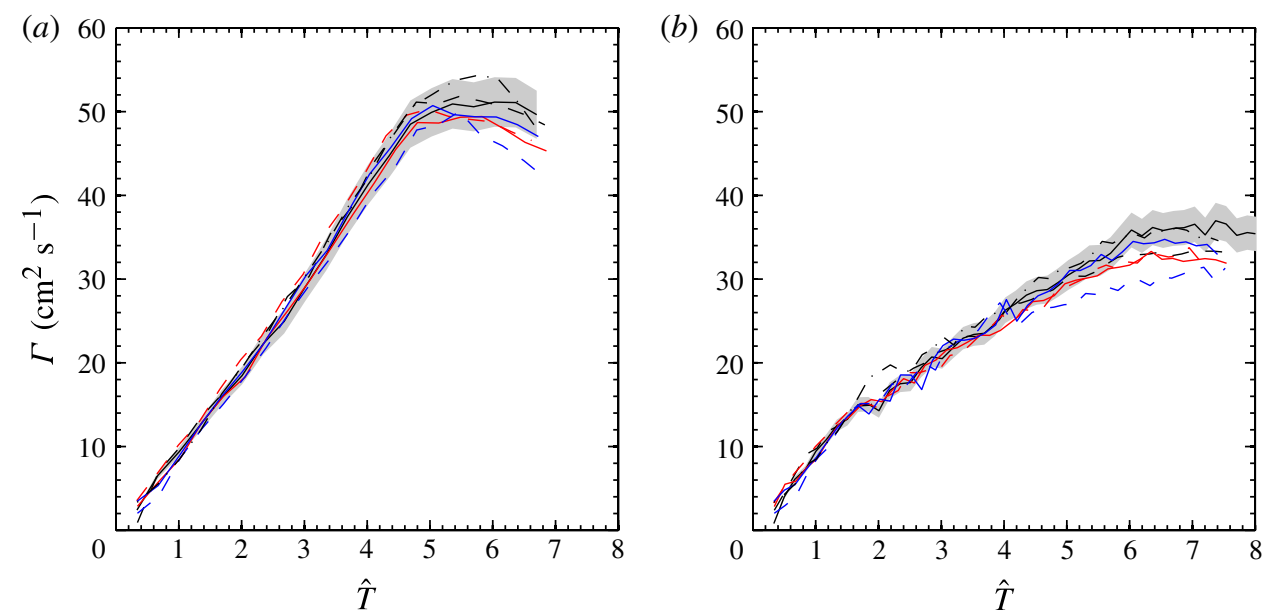

FIGURE 18. Time history of $(a)$ the total circulation emanating from the nozzle and $(b)$ the circulation in the lead vortex, at seven different curvature points on the AR2 nozzle. The error in the measurements was $\approx 6 \%$, and it is shown in grey for the curve on the major axis, for reference. Here $\kappa=1.26 \mathrm{~cm}^{-1}(-) ; \kappa=1.04 \mathrm{~cm}^{-1}(--) ; \kappa=0.82 \mathrm{~cm}^{-1}$ (red line); $\kappa=0.60 \mathrm{~cm}^{-1}$ (dashed red line); $\kappa=0.39 \mathrm{~cm}^{-1}$ (blue line); $\kappa=0.20 \mathrm{~cm}^{-1}$ (dashed blue line); $\kappa=0.16 \mathrm{~cm}^{-1}(-\cdot-)$.

The shaded bands in figure 17 denote the uncertainty in each set of circulation measurements, which was found to be approximately $6 \%$. The uncertainty in the circulation measurements was derived from propagation of the uncertainty in the vorticity measurements, as well as the error associated with defining the area containing the vortex ring and jet. This last quantity was derived from repeated measurements of the total and vortex ring areas, and conservatively estimated to be approximately $10 \%$ of the total area.

Figure 18(a) shows the total circulation as a function of formation time based on nozzle equivalent diameter, for the seven curvature planes along the contour of the AR2 nozzle. In all of the planes considered, the total circulation increased at an almost constant rate until fluid ejection stopped at approximately $\hat{T}=4.8$, when it ceased increasing. The final value of the total circulation in all planes ranged between $\Gamma=48 \mathrm{~cm}^{2} \mathrm{~s}^{-1}$ and $\Gamma=54 \mathrm{~cm}^{2} \mathrm{~s}^{-1}$. Near the minor axis, the shear layer was pushed towards the nozzle centreline by the velocity field induced by the vortex ring. On these planes, vorticity cancellation was evident at longer times, and led to the decrease in the total circulation evident at formation times in excess of $\hat{T}=4.8$ in figure $18(a)$. In figure $18(b)$, we present the time history of the circulation in the leading vortex ring for the seven curvature points. Once again, the seven cases considered exhibited similar trends, with the lead vortex circulation achieving a final value ranging from $\Gamma_{\text {ring }}=31 \mathrm{~cm}^{2} \mathrm{~s}^{-1}$ to $\Gamma_{\text {ring }}=36 \mathrm{~cm}^{2} \mathrm{~s}^{-1}$, at a approximately $\hat{T}=6$.

When computing the lead vortex circulation, we defined the lead vortex core as outlined in $\S 4$. The fact that the circulation within the core as defined above was found to be constant at long formation times confirms our definition of the lead vortex on planes near the minor axis. In contrast, the circulation of the circular portion of the leading vortex on the minor plane continuously increased by drawing circulation from the crescent-shaped vortex, until the breakup of the vortex ring and the formation of the arc-like structure. Hence, although the final circulation in the lead vortex was 

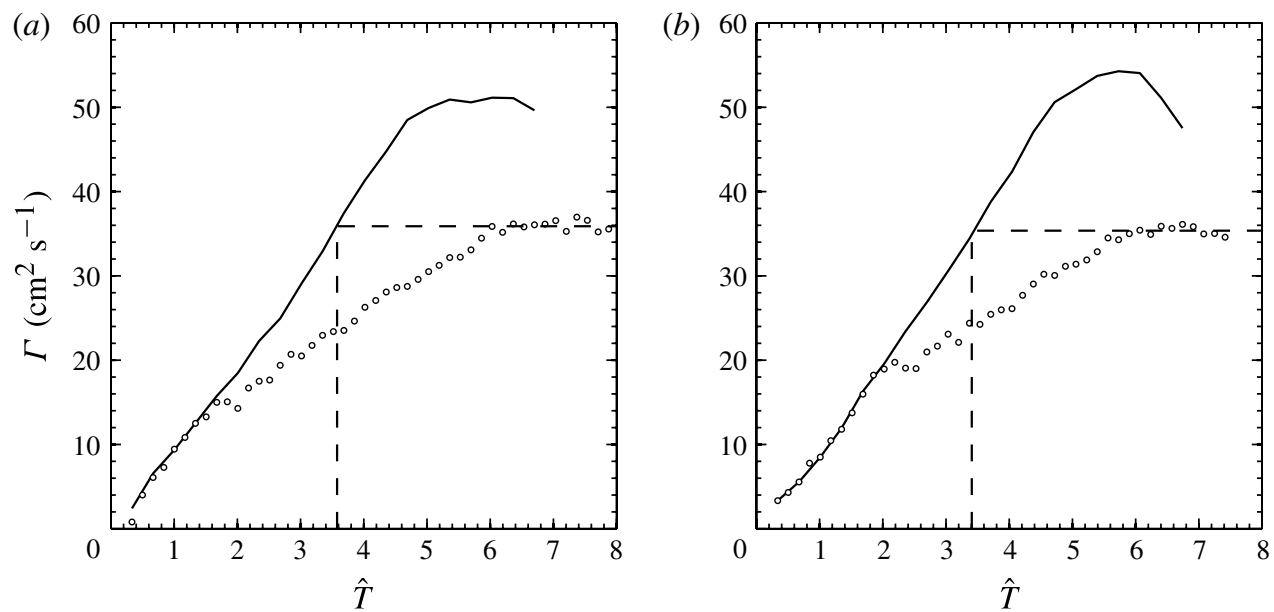

FIGURE 19. Time history of the total circulation (-) and lead vortex circulation (o) on (a) the major axis and $(b)$ the minor axis of the AR2 elliptical nozzle. Comparing the final circulation in the lead vortex with the time history of the total circulation yields the non-dimensional time at which the vortex stopped accepting vorticity $(--)$.

found to be approximately constant across the different curvature planes (figure 18b), the shape of the lead vortex varied significantly between the major axis and the minor axis.

By comparing the time history of the total circulation to the final circulation in the lead vortex, one can determine the non-dimensional time at which the lead vortex ceased to accept additional circulation. We performed this comparison at each data plane, in order to determine whether the formation time at pinch-off varied with the local curvature. Figure 19 illustrates this comparison for two of the seven curvature planes considered: the major and minor axes. On the major axis (figure 19a), the formation time at pinch-off was found to be $\hat{T}=3.6 \pm 0.4$, whereas on the minor axis (figure $19 b$ ) it was found to be $\hat{T}=3.4 \pm 0.4$.

Figure 20 shows the time history of the total circulation emanating from the AR4 elliptical nozzle, as well as the circulation in the lead vortex ring, on the major and minor axes of the nozzle. Comparing the final circulation in the lead vortex ring to the time history of the total circulation yielded a formation time at pinch-off of $\hat{T}=3.1 \pm 0.4$ on the major axis and $\hat{T}=3.5 \pm 0.4$ on the minor axis. Therefore, the formation number based on equivalent diameter for the AR4 nozzle was found to be identical to the formation number for the AR2 nozzle, to within experimental error.

The results in figure 20 are similar to those for the symmetry planes of the AR2 nozzle, with the exception of a step-like increase in the circulation of the lead vortex ring at $\hat{T}=5.5$ in figure $20(a)$, which is absent in figure $19(a)$. In their study of circular vortex rings, Gharib et al. (1998) found that for large stroke ratios, the vortex ring circulation increased in a step-like fashion after long formation times. This step-like increase was caused by the first vortex in the trailing wake catching up to, and coalescing with, the leading vortex ring. This same phenomenon was responsible for the jump in the lead vortex circulation on the major axis in figure 20(a). However, unlike in the circular case, the jump occurred before the lead vortex circulation has levelled off (i.e. before the vortex ring had pinched off). 


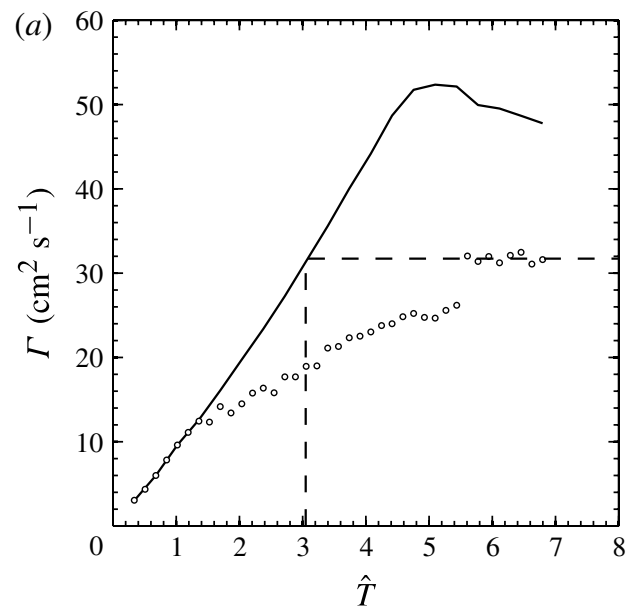

(b)

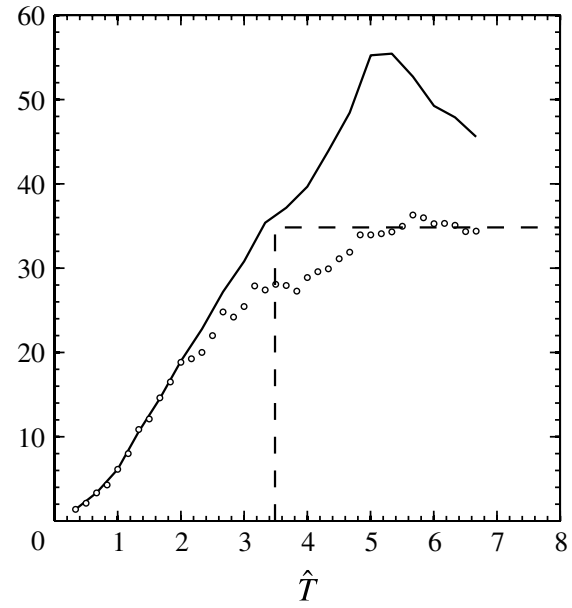

FIGURE 20. Time history of the total circulation (-) and lead vortex circulation (o) on (a) the major axis and $(b)$ the minor axis of the AR4 elliptical nozzle. Comparing the final circulation in the lead vortex with the time history of the total circulation yields the non-dimensional time at which the vortex stopped accepting vorticity $(--)$.
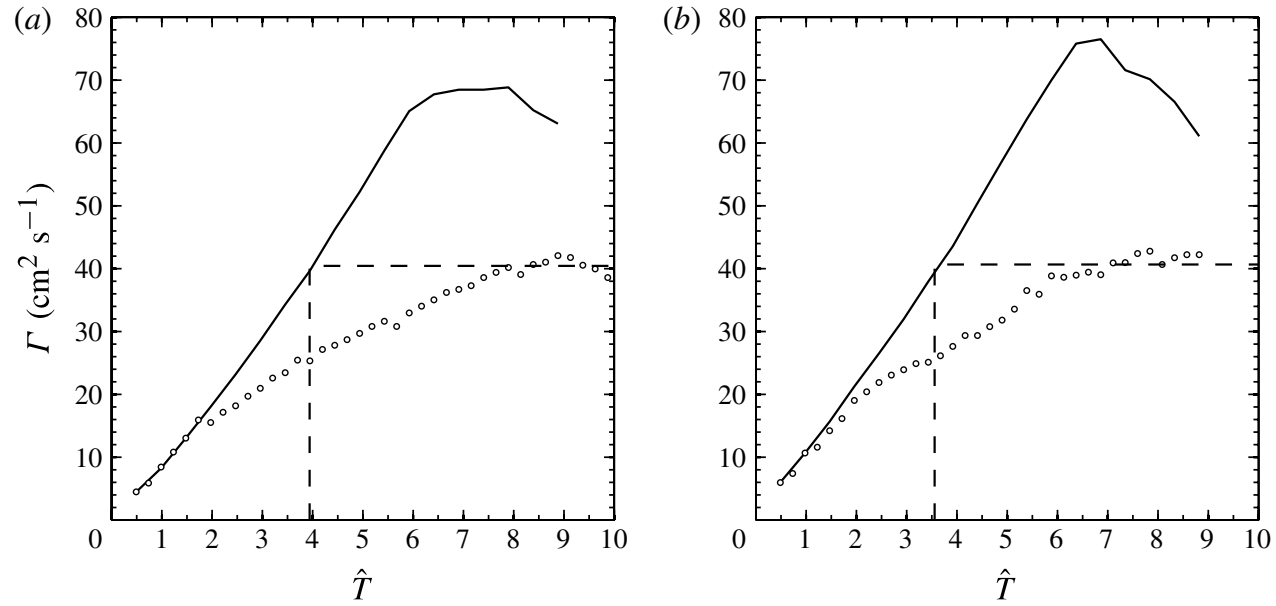

FIGURE 21. Time history of the total circulation (-) and lead vortex circulation (o) on (a) the major axis and $(b)$ the minor axis of the oval nozzle for a stroke ratio of 6.3. Comparing the final circulation in the lead vortex with the time history of the total circulation yields the non-dimensional time at which the vortex stopped accepting vorticity $(--)$.

Figure 21 shows the time history of the total circulation emanating from the oval nozzle, as well as the circulation in the lead vortex ring, on the major and minor axes of the nozzle, for a stroke ratio of 6.3. The circulation time histories for this nozzle were qualitatively very similar to those for the symmetry planes of the two elliptical nozzles, although those cases considered shorter stroke ratios. By comparing the time histories of the vortex ring circulation and the total circulation, pinch-off was found to 


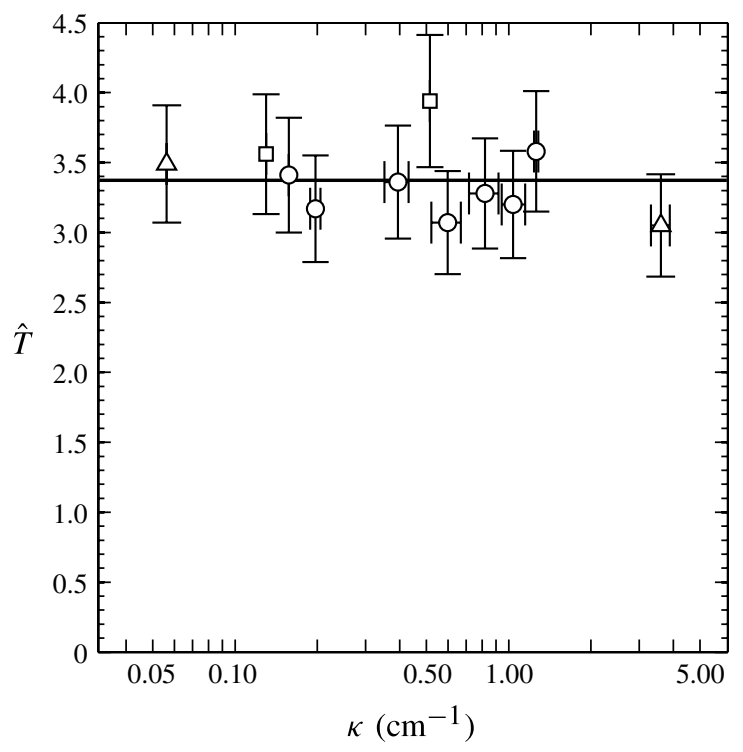

FIgURE 22. Non-dimensional pinch-off time $\left(\hat{T}=\bar{U}_{p} t / D_{e q}\right)$ as a function of curvature for the three nozzle shapes: $\bigcirc$, AR2 nozzle; $\triangle$, AR4 nozzle; $\square$, oval nozzle. The average over all curvature points in the three nozzles $(\hat{T}=3.4 \pm 0.4)$ is denoted by the solid black line.

occur at a formation time of $\hat{T}=3.9 \pm 0.4$ and $\hat{T}=3.6 \pm 0.4$, on the major and minor axes of the oval nozzle, respectively.

Our results are summarized in figure 22, which shows the formation time at pinchoff as a function of curvature, for all three nozzles. The symbols show the formation time at pinch-off at every data plane on the three nozzles and the black line shows the average formation time at pinch-off $(\hat{T}=3.4 \pm 0.4)$, over all data planes. These results suggest that there is no dependence of the formation time at pinch-off on the local curvature, but rather pinch-off occurs simultaneously throughout the nozzle contour, at a formation number based on the equivalent diameter of the nozzle of $\hat{T}=3.4 \pm 0.4$. Moreover, it appears that, for the nozzle shapes considered, the formation number is a function of the equivalent diameter of the nozzle, independent of the nozzle shape. In addition, in all cases, pinch-off was found to occur well before the induced motion of the shear layer towards the nozzle centreline on the minor plane led to axis-touching and localized vorticity cancellation.

\section{Conclusions}

We investigated the formation of vortex rings from three different non-circular nozzles: an elliptical nozzle with an aspect ratio of two, an elliptical nozzle with an aspect ratio of four and an oval nozzle constructed from tangent circular arcs. The nozzles selected encompass a wide class of those non-circular nozzles not considered by Domenichini (2011), since they include nozzles with both smooth and discontinuous variation in the curvature, and span from nozzles with a moderate range of curvatures to those with extreme variations in curvature $\left(\kappa_{\max } / \kappa_{\min }=4\right.$ in the oval nozzle and $\approx 60$ in the AR4 nozzle). 
On all three nozzles, we found that the limiting size and circulation at saturation of the vortex ring were not spatially varying along the local contour and thus not a function of relative variations in the local curvature. Therefore, pinch-off was not found to be a local phenomenon in either of the three nozzle shapes considered. The shape of the leading vortex ring was found to vary along the contour of the nozzle in all three cases and to be strongly dependent on the local curvature. However, the LCS analysis revealed the leading vortices of various shapes to belong to a single coherent leading vortex core during the formation process, thus leading to the observed invariance of the circulation. In addition to the spatial variation in the vortex ring shape, we observed the time-dependent deformation of the vortex, which, in the case of elliptical nozzles, was in agreement with previous studies (Viets \& Sforza 1972; Dhanak \& de Bernardinis 1981; Hussain \& Husain 1989; Adhikari 2009). The deformation of vortex rings from oval nozzles is reported here for the first time, and was found to be qualitatively similar to the deformation of elliptical vortex rings.

In agreement with Hussain \& Husain (1989), we found that the equivalent diameter of a non-circular nozzle is a proper length scale in quantifying the formation of vortex rings with moderate departure from axisymmetry. As a result, we extended the definition of the formation number introduced by Gharib et al. (1998), to vortex rings of this class, using the equivalent diameter of the nozzle as the relevant length scale $\left(\hat{T}=\bar{U}_{p} t / D_{e q}\right)$. In all three nozzles considered, we found the formation number thus defined to be constant along the contour of each nozzle, and to lie in the range between 3 and 4 . This range falls slightly below that observed in circular vortex rings by Gharib et al. (1998) (approximately 3.6-4.5), yet is in remarkably good agreement given the wide class of nozzles considered, and their departure from axisymmetry.

The present results for the shape and deformation of elliptical vortex rings are in good agreement with those of Adhikari (2009). However, our results for the formation number of vortex rings of the three shapes considered contradict Domenichini's conjecture that pinch-off in these geometries should be a local phenomenon governed by the curvature. Given the stark differences between our conclusions about the role of relative curvature in vortex formation, and those of Domenichini (2011), a comparison of the results of the two studies is illuminating.

At the early stages of vortex development, our findings regarding the shape and deformation of the vortex agreed qualitatively with those of Domenichini (2011). Moreover, our results appear compatible with Domenichini's results for the formation of small aspect ratio vortex rings $(h=0.05$ and 0.5 in Domenichini's formulation, where $h$ is the ratio of the length of the rectilinear portion to the diameter of the circular portion). In these cases, Domenichini (2011) found the evolution of the non-circular vortices to be similar to that of circular vortex rings, albeit with some deformation. Furthermore, in these cases, pinch-off appeared to occur simultaneously on the straight and circular sections of the vortex ring.

For larger aspect ratios ( $h=1.5$ and 2 in Domenichini's formulation), the geometry of the vortex ring and shear layer on the symmetry planes was similar in Domenichini's computations and our experimental results. In both studies, a leading vortex ring and trailing shear layer were observed on the major axis plane, while a geometry consisting of a leading vortex ring, crescent vortex and circular vortex core was observed in the minor plane. However, Domenichini (2011) concluded that the circulation in the leading vortex was higher in the flat portions of the vortex than in the circular portions and that pinch-off occurred exclusively on the circular portions of the orifice at a formation time $T^{*}=U t / D=3.6$ based on the diameter of the circular portion of the orifice $(D)$. 
The discrepancy between our results and those of Domenichini's computations for more slender orifices can be attributed, at least in part, to a difference in the definition of the boundaries of the leading vortex on the minor axis between the two studies. Domenichini (2011) presented only results for the circulation on the plane coinciding with the circular portion of one of his pseudo-elliptic orifices $(h=2)$. However, the author kindly granted us access to time-resolved vorticity fields on the two symmetry planes for two of his orifices: one where $h=2$ and one where $h=4$. When computing the circulation of the vortex in both symmetry planes of the $h=2$ orifice using the definition of the vortex boundaries outlined in $\S 4$, we found the circulation in both planes to be similar, indicating that pinch-off occurred simultaneously on both sections of the orifice.

On both axes of the $h=2$ orifice, however, pinch-off appeared to take place at a formation time of 3.6 based on the diameter of the circular portion of the orifice $\left(T^{*}=U t / D\right)$, which corresponded to a formation time of $\hat{T}=1.9$ based on the equivalent diameter of the orifice. Since Domenichini (2011) did not present the time history of the total circulation and vortex ring circulation for orifices other than the $h=2$ case, the relevance of the diameter of the circular segments $(D)$ or the equivalent diameter of the orifice $\left(D_{e q}\right)$ as length scales for Domenichini's flows cannot be ascertained.

In addition, the crescent-shaped vortex observed in the minor plane in both our experiments and those of Adhikari (2009) was not found in the vorticity data for Domenichini's most slender orifices $(h=4)$. In this case, the shear layer was found to curve around the leading vortex, but no separation of the crescent-shaped portion of the shear layer from its main section was observed. The absence of the crescent vortex might be indicative of a change in the vortex formation process for very-high-aspectratio orifices. Such a change is consistent with the two-dimensional rollup observed in the limiting case of extremely thin slits (Afanasyev 2006; Pedrizzetti 2010).

The planar DPIV method utilized in this study allows for the reconstruction of an approximate three-dimensional vorticity field only for vortex rings with relatively small departures from axisymmetry. In order to analyse the three-dimensional dynamics of vortex rings such as the oval vortex ring and AR4 elliptical vortex ring considered in this study, three-dimensional velocimetry is required. The three-dimensional, threecomponent defocusing digital particle image velocimetry (DDPIV) technique (Willert \& Gharib 1992; Pereira \& Gharib 2002; Pereira et al. 2006) has been applied successfully to non-axisymmetric vortex ring flows by Troolin \& Longmire (2009) and Kim \& Gharib (2011), and is a promising candidate for performing three-dimensional velocimetry measurements on vortex rigs undergoing large deformations.

The results presented in this study covered a class of vortex rings with moderate departure from axisymmetry, which is representative of many vortex rings of biological relevance. However, at present the extent to which our results extend to vortex rings outside of this class is unknown. In addition, the limitations of our results in terms of aspect ratio and nozzle geometry are also unknown. Given the differences in the formation number between the present results and those of Domenichini (2011), determining the extent to which the equivalent diameter is the relevant length scale in defining a global formation number is of interest. Further studies on the formation of vortices outside of the biologically relevant class could enable the definition of a (possibly locally varying) analogue to the formation number of Gharib et al. (1998), which extends to a large class of vortex ring geometries and recovers the asymptotic behaviour of circular vortex rings and two-dimensional vortex dipoles. 
The present results have potentially far-reaching implications for the study and modelling of vortex ring flows of biological relevance. Elliptical, elongated and otherwise non-circular vortex rings such as those investigated here are known to be a distinguishing feature in many biological flows (Bellhouse 1972; Kokshaysky 1979; Dickinson \& Götz 1996; Domenichini et al. 2005; Kern \& Koumoutsakos 2006). Our results on the formation number of vortex rings of this class therefore allow for a more accurate understanding of the parameters governing the formation of vortex rings in the human left ventricle, for example.

Furthermore, the concepts of the vortex formation number and optimal vortex formation have been applied successfully to evaluating the efficiency or optimality of propulsive flows (Krueger \& Gharib 2003; Linden \& Turner 2004; Dabiri et al. 2010) which feature vortex rings of almost circular shape. However, in other locomotory flows where non-axisymmetric vortex rings are the norm, the absence of known limits on vortex ring size previously precluded this sort of analysis. The present results, however, suggest that we can reduce vortex rings with moderate departure from axisymmetry to circular vortex rings with the same equivalent diameter. Such a reduction allows for greater ease of modelling, and a decrease in computational complexity in numerical studies, through the exploitation of axisymmetry. Simplifications of this kind have been perfumed in the past by researchers studying both cardiac (Steen \& Steen 1994; Gharib et al. 2006; Kheradvar \& Gharib 2007) and locomotory flows (Drucker \& Lauder 1999; Dickinson et al. 2000; Hsieh \& Lauder 2004).

\section{Acknowledgements}

This work was funded by an NSF Graduate Research Fellowship to C.O'F., and by Office of Naval Research awards N000140810918 and N000141010137 to J.O.D. The authors gratefully acknowledge the contributions of R.W. Whittlesey, who was responsible for the design of the AR4 nozzle, and who assisted in the design and construction of the experimental setup.

\section{REFERENCES}

ADHIKARI, D. 2009 Some experimental studies on vortex ring formation and interaction. Master's thesis, National University of Singapore, available online at http://scholarbank.nus.edu.sg/ handle/10635/16569.

AdRIAN, R. J. 1991 Particle-imaging techniques for experimental fluid mechanics. Annu. Rev. Fluid Mech. 23, 261-304.

Afanasyev, Y. D. 2006 Formation of vortex dipoles. Phys. Fluids 18, 037103.

Ahuja, A., Manes, J. \& MAssey, K. 1990 An evaluation of various concepts of reducing jet noise. AIAA Paper 90-3982.

ARMS, R. J. \& HAMA, F. R. 1965 Localized-induction concept on a curved vortex and motion of an elliptic vortex ring. Phys. Fluids 8 (4), 553-559.

AuErbach, D. 1991 Stirring properties of vortex rings. Phys. Fluids A 3, 1351-1355.

BAiRd, M. H. I., WAiregi, T. \& LoO, H. J. 1977 Velocity and momentum of vortex rings in relation to formation parameters. Can. J. Chem. Engng 55, 19-26.

Bartol, I. K., Krueger, P. S., Stewart, W. J. \& Thompson, J. T. 2009 Pulsed jet dynamics of squid hatchlings at intermediate Reynolds numbers. J. Exp. Biol. 212, 1506-1518.

Bellhouse, B. J. 1972 Fluid mechanics of a model mitral valve and left ventricle. Cardiovasc. Res. 6, 199-210. 
Benjamin, T. B. 1976 The alliance of practical and analytical insights into the nonlinear problems of fluid mechanics. In Applications of Methods of Functional Analysis to Problems in Mechanics (ed. P. Germain \& B. Nayroles), pp. 8-28. Springer.

Colin, S. P. \& Costello, J. H. 2002 Morphology, swimming performance and propulsive mode of six co-occurring hydromedusae. J. Exp. Biol. 205, 427-437.

Dabiri, J. O., Colin, S. P., Katija, K. \& Costello, J. H. 2010 A wake-based correlate of swimming performance and foraging behaviour in seven co-occurring jellyfish species. J. Exp. Biol. 213, 1217-1225.

DABIRI, J. O. \& GHARIB, M. 2004a Delay of vortex ring pinch-off by an imposed bulk counerflow. Phys. Fluids 16 (L), 28-30.

DABIRI, J. O. \& GHARIB, M. 2004b Fluid entrainment by isolated vortex rings. J. Fluid Mech. 511, $311-331$.

DABIRI, J. O. \& GHARIB, M. 2005 The role of optimal vortex formation in biological fluid transport. Proc. R. Soc. B 272, 1557-1560.

DhAnaK, M. R. \& DE Bernardinis, B. 1981 The evolution of an elliptic vortex ring. J. Fluid Mech. 109, 189-216.

Dickinson, M. H., Farley, C. T., Full, R. J., Koehl, M. A. R., Kram, R. \& Lehman, S. 2000 How animals move: an integrative view. Science 288 (5463), 100-106.

Dickinson, M. H. \& GöTZ, K. G. 1996 The wake dynamics and flight forces of the fruit fly Drosophila melanogaster. J. Exp. Biol. 199, 2085-2104.

DIDDEN, N. 1979 On the formation of vortex rings: rolling-up and production of circulation. Z. Angew. Math. Phys. 30, 101-116.

Domenichini, F. 2011 Three-dimensional impulsive vortex formation from slender orifices. J. Fluid Mech. 666, 506-520.

Domenichini, F., Pedrizzetti, G. \& Baccani, B. 2005 Three-dimensional filling flow into a model left ventricle. J. Fluid Mech. 539, 179-198.

DRUCKER, E. G. \& LAUDER, G. V. 1999 Locomotor forces on a swimming fish: three-dimensional vortex wake dynamics quantified using digital particle image velocimetry. J. Exp. Biol. 202, 2393-2412.

Du ToIT, P. C. 2010 Transport and separatrices in time-dependent flows. PhD thesis, California Institute of Technology.

Fernandez, V. M., ZABUSKy, N. J. \& GRYANiK, V. M. 1995 Vortex intensification and collapse of the Lissajous-elliptic ring: single- and multi-filament Biot-Savart simulations and visiometrics. J. Fluid Mech. 299, 289-331.

Gan, L., Dawson, J. R. \& Nickels, T. B. 2012 On the drag of turbulent vortex rings. J. Fluid Mech. 709, 85-105.

Gharib, M., Rambod, E., Kheradvar, A., Sahn, D. J. \& Dabiri, J. O. 2006 Optimal vortex formation as an index for cardiac health. Proc. Natl Acad. Sci. USA 103, 6305-6308.

GHARIB, M., RAmbod, E. \& ShARIFF, K. 1998 A universal time scale for vortex ring formation. J. Fluid Mech. 360, 121-140.

GlADFElter, W. G. 1973 A comparative analysis of the locomotory systems of medusoid Cnidaria. Helgol. Wiss. Meeresunters 25, 228-272.

Glezer, A. \& Coles, D. 1990 An experimental study of a turbulent vortex ring. J. Fluid Mech. 221, 243-283.

Green, M. A, Rowley, C. W. \& Haller, G. 2007 Detection of Lagrangian coherent structures in 3D turbulence. J. Fluid Mech. 572, 111-120.

Green, M. A, Rowley, C. W. \& Smits, A. J. 2010 Using hyperbolic Lagrangian coherent structures to investigate vortices in bio-inspired fluid flows. Chaos 20, 017510.

GRInstein, F. F. 1995 Self-induced vortex ring dynamics in subsonic rectangular jets. Phys. Fluids 7 (10), 2519-2521.

Grinstein, F. F. 2001 Vortex dynamics and entrainment in rectangular free jets. J. Fluid Mech. 437, 69-101.

Grinstein, F. F., Glauser, M. N. \& George, W. K. 1995 Vorticity in jets. In Fluid Vortices (ed. S. I. Green), Kluwer Academic.

GutMARK, E. J. \& GRInStein, F. F. 1999 Flow control with noncircular jets. Annu. Rev. Fluid Mech. 31, 239-272. 
HAller, G. 2000 Finding finite-time invariant manifolds in two-dimensional velocity fields. Chaos 10, 99.

HALleR, G. 2001a Distinguished material surfaces and coherent structures in three-dimensional flows. Physica D 149, 1851-1861.

HALlER, G. $2001 b$ Lagrangian structures and the rate of strain in a partition of two-dimensional turbulence. Phys. Fluids 13, 3365-3385.

Haller, G. 2002 Lagrangian coherent structures for approximate velocity data. Phys. Fluids 14 (6), 1851-1861.

Haller, G. \& Poje, A. C. 1998 Finite time transport in aperiodic flows. Physica D 119, $352-380$.

Haller, G. \& YuAN, G. 2000 Lagrangian coherent structures and mixing in two-dimensional turbulence. Physica D 147, 352-370.

Ho, C.-M. \& GUTMARK, E. 1987 Vortex induction and mass entrainment in a small-aspect-ratio jet. J. Fluid Mech. 179, 383-405.

Hsieh, S. T. \& LAuder, G. V. 2004 Running on water: three-dimensional force generation by basilisk lizards. Proc. Natl Acad. Sci. USA 101 (48), 16784-16788.

Husain, H. S. \& Hussain, F. 1993 Elliptic jets. Part 3. Dynamics of preferred mode coherent structure. J. Fluid Mech. 248, 315-361.

Hussain, F. \& Husain, H. S. 1989 Elliptic jets. Part 1. Characteristics of unexcited and excited jets. J. Fluid Mech. 208, 257-320.

Hussain, H. S. \& Hussain, F. 1991 Elliptic jets. Part 2. Dynamics of coherent structures: pairing. J. Fluid Mech. 233, 439-482.

Kambe, T. \& TAkao, T. 1971 Motion of distorted vortex rings. J. Phys. Soc. Japan 31 (2), 591-599.

Kelvin, Lord 1880 Vortex statics. Phil. Mag. 10, 97-109.

Kern, S. \& Koumoutsakos, P. 2006 Simulations of optimized anguilliform swimming. J. Exp. Biol. 209, 4841-4857.

KHERADVAR, A. \& GHARIB, M. 2007 Influence of ventricular pressure drop on mitral annulus dynamics through the process of vortex ring formation. Ann. Biomed. Engng 35 (12), 2050-2064.

KIM, D. \& GHARIB, M. 2011 Flexibility effects on vortex formation of translating plates. J. Fluid Mech. 677, 255-271.

KIMURA, Y. 2006 Motion of an elliptic vortex ring and particle transport. In IUTAM Symposium on Hamiltonian Dynamics, Vortex Structures, Turbulence (ed. A. V. Borisov, V. V. Kozlov, I. S. Mamaev \& M. A. Sokolovskiy), pp. 119-124. Springer.

KIYA, M. \& IsHiI, H. 1991 Deformation and splitting of pseudo-elliptic vortex rings. In Advances in Turbulence 3 (ed. A. V. Johansson \& P. H. Alfredsson), pp. 52-60. Springer.

Kiya, M., Toyoda, K., Ishit, H., Kitamura, M. \& OHe, T. 1992 Numerical simulation and flow-visualization experiments on deformation of pseudo-elliptic vortex rings. Fluid Dyn. Res. 10, 117-131.

KOKSHAYSKY, N. V. 1979 Tracing the wake of a flying bird. Nature 279, 146-148.

Krueger, P. S., DABIRI, J. O. \& GHARIB, M. 2006 The formation number of vortex rings in uniform background coflow. J. Fluid Mech. 556, 147-166.

Krueger, P. S. \& GHarib, M. 2003 The significance of vortex ring formation to the impulse and thrust of a starting jet. Phys. Fluids 15, 1271-1281.

LEONARD, A. 1985 Computing three-dimensional incompressible flows with vortex elements. Annu. Rev. Fluid Mech. 17, 523-559.

Linden, P. F. \& TURner, J. S. 2004 'Optimal' vortex rings and aquatic propulsion mechanisms. Proc. R. Soc. B 271, 647-653.

Maxworthy, T. 1972 The structure and stability of vortex rings. J. Fluid Mech. 51 (1), 15-32.

Mohseni, K., Ran, H. \& ColoniUs, T. 2000 Numerical experiments on vortex ring formation. J. Fluid Mech. 430, 267-282.

O’Dor, R. K. 1988 The forces acting on swimming squid. J. Exp. Biol. 137, 421-442.

O'FARrell, C. \& DABiri, J. O. 2010 A Lagrangian approach to vortex pinch-off. Chaos 20, 017513. 
Olcay, A. B. \& Krueger, P. S. 2007 Measurements of ambient fluid entrainment during laminar vortex formation. Exp. Fluids 44, 235-247.

Olcay, A. B. \& Krueger, P. S. 2010 Momentum evolution of ejected and entrained fluid during laminar vortex ring formation. Theor. Comput. Fluid Dyn. 24, 465-482.

Oshima, Y. 1972 Motion of vortex rings in water. J. Phys. Soc. Japan 32 (4), 1125-1131.

Oshima, Y., Izutsu, N., Oshima, K. \& Hussain, A. K. M. F. 1988 Bifurcation of an elliptic vortex ring. Fluid Dyn. Res. 3, 133-139.

Pawlak, G., Cruz, C. M., Bazan, C. M. \& Hrdy, P. G. 2007 Experimental characterization of starting jet dynamics. Fluid Dyn. Res. 39, 711-730.

Pedrizzetti, G. 2010 Vortex formation out of two-dimensional orifices. J. Fluid Mech. 655, $198-216$.

PenG, J. \& DABIRI, J. O. 2009 Transport of inertial particles by Lagrangian coherent structures: application to predator-prey interactions in jellyfish feeding. J. Fluid Mech. 623, 75-84.

Pereira, F. \& GHARIB, M. 2002 Defocusing digital particle image velocimetry and the three-dimensional characterization of two-phase flows. Meas. Sci. Technol. 13, 683-694.

Pereira, F., Heinrich, S., GrafF, E. C. \& Gharib, M. 2006 Two-frame 3D particle tracking. Meas. Sci. Technol. 17, 1680-1692.

RAYNer, J. M. V. 1979 A vortex theory of animal flight. Part 2. The forward flight of birds. J. Fluid Mech. 91, 731-763.

Reul, H., TAlukder, N. \& Muller, W. 1981 Fluid mechanics of the natural mitral valve. J. Biomech. 14, 361-372.

Rosenfeld, M., RAMBOD, E. \& GHARIB, M. 1998 Circulation and formation number of laminar vortex rings. J. Fluid Mech. 376, 297-318.

RYU, K.-W. \& LEE, D.-J. 1997 Sound radiation from elliptic vortex rings: evolution and interaction. J. Sound Vib. 200 (3), 281-301.

Saffman, P. G. 1992 Vortex Dynamics. Cambridge University Press.

Shadden, S. C., Dabiri, J. O. \& MARsden, J. E. 2006 Lagrangian analysis of fluid transport in empirical vortex ring flows. Phys. Fluids 18, 047105.

Shadden, S. C., Katija, K., Rosenfeld, M., Marsden, J. E. \& Dabiri, J. O. 2007 Transport and stirring induced by vortex formation. J. Fluid Mech. 593, 315-331.

Steen, T. \& SteEn, S. 1994 Filling of a model left ventricle studied by colour M mode Doppler. Cardiovas. Res. 28, 1821-1827.

Troolin, D. R. \& LongmiRe, E. K. 2009 Volumetric velocity measurements of vortex rings from inclined exits. Exp. Fluids 48, 409-420.

Viets, H. \& Sforza, P. M. 1972 Dynamics of bilateraly symmetric vortex rings. Phys. Fluids 13 (2), 230-240.

WeIHS, D. 1977 Periodic jet propulsion of aquatic creatures. Fortschr. Zool. 24, 171-175.

Wieting, D. W. \& Stripling, T. E. 1984 Dynamics and fluid dynamics of the mitral valve. In Recent Progress in Mitral Valve Disease (ed. C. Duran, W. W. Angell, A. D. Johnson \& J. H. Oury), pp. 13-46. Butterworths.

Willert, C. E. \& Gharib, M. 1991 Digital particle image velocimetry. Exp. Fluids 10, 181-193.

Willert, C. E. \& GHARIB, M. 1992 Three-dimensional particle imaging with a single camera. Exp. Fluids 12, 353-358.

Zhao, W., Frankel, S. H. \& Mongeau, L. G. 2000 Effects of trailing jet instability on vortex ring formation. Phys. Fluids 12, 589-621. 